Apidologie, 1971, 2 (2), 123-155.

\title{
L'INFLUENCE DE LA REINE D'ABEILLE \\ (APIS MELLIFICA L.) \\ SUR LA PRISE DE NOURRITURES DES OUVRIẼRES ACCOMPAGNATRICES
}

\author{
Der Einfluss der Bienenkönigin (Apis mellifica L.) \\ auf die Nahrungsaufnahme der Begleitbienen
}

Bernard ROGER

Station de Recherches sur l'Abeille et les Insectes Sociaux, I.N.R.A., 91 - Bures-sur-Yvette

\section{SUMMARY}

\author{
THE QUEEN BEES' INFLUENCE ON THE FOOD INTAKE OF THE \\ ATTENDANT WORKER BEES
}

The author highlights the interaction between the queen bees and the worker bees by investigating the food intake of young bees of known age.

Such intakes are assessed by weighing the food daily during the first seven days for the pollen and throughout the test, i.e., 15 days, for candy sugar.

There are two significant periods for the candy sugar intake, i.e., from the lst to the 7 th, and from the 8th to the 15 th day. Over the lst period the data evinced actual consumption. On the following days, it was found that candy sugar was converted into syrup stored at the bottom of beautifully formed wax cells.

The young queens, whether virgin or fertilized, promote a sweet food intake for the attendant bees. A statistical analysis corroborates this fact. Conversely, in presence of ageing queens, the workers take less candy sugar than do the orphan bees.

It is always the latter which consume the most pollen.

The queen bees promote the intake of sweet food and do this more or less markedly, depending on their age and on the hatching time of their attendant bees. The same factors affect the building capability of these workers.

The physiological condition of the bees is apparently of some importance. 


\section{RESUMÉ}

L'auteur met en évidence les interactions entre reine et ouvrières par l'étude de la prise de nourriture chez les jeunes abeilles d'âge connu.

Les prélèvements sont évalués par la pesée quotidienne des aliments, pendant les 7 premiers jours pour le pollen et sur l'ensemble de l'expérience, c'est-à-dire 15 jours, pour le candi.

On distingue deux périodes essentielles dans le prélèvement de candi : du $I^{\mathrm{er}}$ au $7^{\mathrm{e}}$ jour et du $8^{\mathrm{e}}$ au $15^{\mathrm{e}}$ jour. Au cours de la première période, les chiffres traduisent une consommation réelle. Les jours suivants on constate la transformation du candi en sirop et son stockage au fond de cellules de cire parfaitement étirées.

Les jeunes reines, vierges aussi bien que fécondes, favorisent la prise de nourriture sucrée des abeilles accompagnatrices. Les résultats sont confirmés par l'analyse statistique. Par contre, en présence de reines vieillies, les ouvrières prélèvent moins de candi que les orphelines.

Ce sont toujours les abeilles orphelines qui consomment le plus de pollen. Les reines stimulent la prise de nourriture sucrée et semblent exercer cette influence de manière plus ou moins marquée, d'une part selon leur état de vieillissement et d'autre part selon l'époque d'éclosion de leurs abeilles accompagnatrices. Les mêmes facteurs influent sur l'aptitude à la construction de ces ouvrières.

L'importance de l'état physiologique des abeilles ne semble pas négligeable.

\section{INTRODUCTION}

Nous avions entrevu au cours d'expériences précédentes (Roger et Pain, 1966) l'intérêt qu'il y aurait à étudier la prise de nourriture de l'abeille encagée, d'une part lorsqu'elle est orpheline, et par conséquent placée dans des conditions biologiques anormales, $d$ 'autre part, lorsque cette abeille, en présence de reine, retrouve en partie les conditions d'une ruche normale. DenvertSalleron (1963), à la suite d'une étude sur les échanges de nourriture entre reine et ouvrières, considère que le groupe d'abeilles d'une cagette expérimentale peut-être comparé à une colonie en réduction avec une organisation sociale similaire.

Malgré l'importance des travaux se rapportant à l'alimentation des ouvrières d'abeilles claustrées, les interactions reines-ouvrières dans cette situation particulière ne semblent pas avoir interessé jusqu'ici les auteurs. PaIn (1961, 1963) a fait une mise au point bibliographique très complète sur l'alimentation des ouvrières d'abeilles. C'est la raison pour laquelle nous nous sommes limité ici aux travaux dont les données sont quantifiées et peuvent être ainsi comparées aux nôtres.

Les recherches effectuées sur les ouvrières maintenues dans une étuve traitent surtout de l'action de la nourriture sur la durée de vie des abeilles, le développement des principales glandes céphaliques, les ovaires, les réserves graisseuses, enfin sur les variations de poids et la teneur en azote. Elles portent aussi sur l'effet de groupe. Au cours de ces recherches l'évaluation des quantités d'aliments ingérés est considérée comme de moindre importance par rapport aux effets physiologiques précédemment cités. 
D'autre part, il est assez difficile d'établir une comparaison précise entre toutes les données relatives à la consommation des diverses nourritures par les abeilles. Fn effet, les auteurs n'appliquent pas les mêmes méthodes expérimentales. Les principales variations portent sur l'âge des abeilles employées la pluralité et la qualité des nourritures, leur mode de présentation.

MaUrizio $(1946,1958)$ a montré qu'il existait un rapport étroit entre la longévité des abeilles claustrées et le développement des corps gras, des glandes céphaliques et des ovaires. Elle mesure la nourriture consommée (1946). Elle alimente les abeilles avec, soit du candi seul, soit du candi auquel elle ajoute du pollen dans des proportions variables. Elle conclut que la prise de nourriture est fonction de l'âge des abeilles et dépend aussi de la saison au cours de laquelle celles-ci sont utilisées. Elle note que les jeunes abeilles consomment moins de nourriture que les abeilles plus âgées prélevées au hasard dans les ruches.

De Groot (1953), parvient aux mêmes conclusions. Selon lui, sur les mêmes régimes, les vieilles abeilles consomment beaucoup plus de nourriture que les jeunes de 4 à 5 jours. Toutefois l'importance des prélèvements en début d'expérience est plus marquée et plus régulière avec les jeunes abeilles. Plus les abeilles mangent et plus grande est leur longévité. Par ailleurs, il constate de très grandes variations journalières dans la prise de nourriture.

Les résultats très complets des expériences de WAHL (1963), effectuées avec de jeunes abeilles orphelines, montrent que la consommation est en général plus forte pendant la $1^{\text {ere }}$ semaine, puis qu'elle diminue nettement au cours de la $2^{e}$ et de la $3^{e}$ semaine, pour devenir minimale ensuite, quel que soit l'aliment considéré.

Pain (1961, 1963), étudiant plus spécialement la consommation de pollen, trouve que celle-ci est très importante pendant les 4 à 5 premiers jours de captivité des jeunes abeilles, puis qu'elle diminue pour devenir nulle vers le 10 jour. Inversement, la consommation en sucre augmente progressivement jusqu'à ce même $10^{\mathrm{e}}$ jour.

PaIN (1961), en présentant les nourritures de façon séparée s'est placée plus près des conditions naturelles. Cette méthode permet une meilleure interprétation des consommations et facilite l'étude de la nutrition de l'abeille.

Pain et Maugenet (1966), étudient comparativement l'appétence du pollen frais et du pollen fermenté et son influence sur l'ovogénèse des ouvrières. Le pollen frais serait mieux consommé que le pollen fermenté.

Dans un travail plus récent portant sur l'effet de groupe chez l'abeille, Sitbon (1967), est amené à effectuer des mesures de consommation. Il constate que les abeilles isolées meurent plus vite que les abeilles groupées par 50 . Cette mortalité ne serait pas due à un refus de nourriture mais à l'isolement 
lui-même. Les abeilles isolées ou groupées consommeraient sensiblement autant. Toutefois, la consommation des abeilles groupées serait plus importante que celle des isolées pendant les 4 premiers jours.

A la lumière de ces travaux, il apparaît que les premiers jours de vie de l'abeille claustrée ont une importance capitale en ce qui concerne la consommation de nourriture, quelle qu'elle soit.

D'autre part nous savons que c la prise de nourriture est un fait de comportement, premier maillon de la chaîne des phénomènes dont l'ensemble constitue la fonction de nutrition ». (J.M. LEgaY, 1957). Or, l'âge et la température affectent quantitativement la prise de nourriture de l'abeille. En introduisant une reine au sein de petites populations encagées, le comportement alimentaire des abeilles accompagnatrices se trouve-t-il modifié, dans quel sens et pour quelle nourriture?

C'est ce que nous avons essayé d'analyser au cours de 2 années d'expérimentation.

\section{MATÉRIEL ET MÉTHODE}

\section{1. - Matériel biologique}

\section{Les ouvrières.}

Nous avons utilisé uniquement de jeunes abeilles d'âge connu, nées en étuve à la température de $32^{\circ} \mathrm{C}$. - Pour les expériences $\mathrm{n}^{\mathrm{os}} 1,2,4,5,6$, les cadres de couvain proviennent de ruches situées à l'extérieur.

Pour les expériences $n^{\text {os }} 3$ et 7 les cadres de couvain ont été prélevés dans des colonies maintenues dans un rucher couvert à une température ambiante de $20^{\circ} \mathrm{C}$. Ces dernières colonies reçoivent quotidiennement $100 \mathrm{~cm}^{3}$ de sirop de sucre tiède à $50 \%$, de l'eau à volonté et 2 fois par mois environ $300 \mathrm{~g}$ de pollen (conservé non séché à $-24{ }^{\circ} \mathrm{C}$.) distribué humecté de miel dans les cellules d'un rayon.

\section{Les reines.}

L'introduction des reines à l'intérieur des cagettes expérimentales s'est toujours déroulée selon le même processus. Les reines sont badigeonnées d'eau miellée à l'aide d'un fin pinceau. Elles sont ensuite délicatement déposées au sein d'une petite population de jeunes abeilles; celles-ci ne manifestent aucune agressivité envers la reine. De cette manière l'acceptation est toujours bonne et rapide.

a) Reines vierges.

Les reines vierges dont nous nous sommes servi sont nées en étuve. Elles sont issues d'un élevage réalisé à la Station de Bures-sur-Yvette à partir de colonies d'abeilles italiennes. (Apis mellifica ligustica Spinola).

Avant leur emploi à des fins expérimentales, elles sont réparties à l'intérieur de petits groupes de 40 à 50 jeunes abeilles d'âge connu, dans des cagettes d'attente.

b) Reines fécondes.

Après un séjour d'au moins 6 semaines en ruchettes 3 cadres, les reines ont été prélevées, après vérification de leur ponte, afin de servir aux expériences $n^{\mathrm{os}} 3,4,5$.

c) Reines vieillies en étuve (vierges et fécondes).

Les reines vierges et fécondes ont été vieillies de la même façon. Nous les avons maintenues sur candi et pollen, encagées avec des groupes de 50 abeilles renouvelées tous les mois jusqu'au moment de leur utilisation. 


\section{Matériel technique}

Pour toutes nos expériences, nous avons employé le modèle de cagette en Altuglass mis au point par J. PaIN (1966).

Ces cagettes étaient maintenues en étuve à la température de $32^{\circ} \mathrm{C}$. Pour plus de commodité, nous avons remplacé les mangeoires d'origine par des mangeoires en polystyrène de forme parallélépipédique et de dimensions : $21 \times 21 \times 7 \mathrm{~mm}$, pouvant contenir environ $5 \mathrm{~g}$. de candi.

Pour la distribution du pollen, nous nous sommes servi de petites mangeoires cylindriques en plastique : $\varnothing 14 \mathrm{~mm}$, hauteur : $9 \mathrm{~mm}$, d'une capacité d'environ $1,6 \mathrm{~g}$.

Le toit des cagettes était muni d'une languette de cire gaufrée.

\section{3. - Méthode}

Notre méthode de répartition des abeilles au hasard dans les cagettes a été décrite dans une publication précédente (Roger, PaIN, 1966). Elle nous permet d'obtenir un matériel biologique homogène.

Chaque expérience comportait un minimum de 2 lots de 6 cagettes. Chacune de celles-ci contenait le même nombre d'ouvrières d'âge identique. Les populations de l'un des 2 lots restaient orphelines (témoins) les autres recevaient une reine (traitées).

Les mortalités étaient notées chaque jour et les cagettes maintenues propres tout au long des expériences.

\section{4. - Les aliments, appréciation des prises de nourritures}

Nous avons choisi pour nos différentes expériences, de nourrir les abeilles avec du candi et du pollen. L'eau était donnée ad libitum.

Le candi se présente sous forme de pâte maléable. Il est composé de miel et de sucre glace dans la proportion de $1: 3$.

Le pollen était administré seul, à part du candi, selon la méthode de Pain (1961). Il provenait d'un même stock constitué par un mélange de récoltes; celles-ci étaient obtenues grâce à des trappes à pollen posées sur quelques ruches au cours de la saison 1966 dans la région parisienne. Il était conservé non séché, dans un appareil frigorifique à la température de $-24^{\circ} \mathrm{C}$. Les principaux pollens constitutifs du mélange utilisé étaient : saules, arbres fruitiers, Crucifères, châtaignier et trèfles.

Le candi et le pollen étaient renouvelés chaque jour. Chaque mangeoire et chaque cagette étaient numérotées. Les pesées étaient effectuées quotidiennement à la même heure, pendant 15 jours pour le candi, et pendant les 7 premiers jours seulement pour le pollen. En effet, les abeilles ne consomment pratiquement plus de pollen au-delà du $7^{\mathrm{e}}$ jour.

Les mangeoires étaient enlevées toutes en même temps selon un ordre préétabli. Après chaque pesée, nous les replaçions dans le même ordre.

Une fois les nourritures distribuées, nous faisions varier la disposition des cagettes à l'intérieur de l'étuve.

L'ensemble des pesées duraient de 45 à 90 minutes environ, en fonction du nombre de cagettes employées pour les expériences. Les manipulations étant assez longues comme nous venons de l'indiquer, et pour éviter de perturber le comportement alimentaire, nous avons effectué les pesées de l'ensemble des mangeoires de chacun des 2 lots.

Afin d'apprécier la dessication des aliments sucrés et azotés, nous disposions pour ehaque expérience de 2 cagettes sans abeilles, mais munies d'un abreuvoir rempli d'eau et contenant 2 mangeoires, l'une avec candi, l'autre avec pollen. Ces deux nourritures étaient renouvelées et pesées quotidiennement. Les cagettes de contrôle étaient intercalées entre les cagettes peuplées.

Nous pensons que les jeunes abeilles subissent un léger “ stress 》 lors de l'encagement. Afin d'atténuer celui-ci, nous avons utilisé les abeilles 15 à $18 \mathrm{~h}$ après leur encagement en procédant de la façon suivante :

en fin d'après-midi, nous encagions les jeunes abeilles naissantes. Environ 2 à 3 heures plus tard, nous introduisions les reines destinées à l'expérimentation dans une partie des cagettes. Les abeilles se trouvaient alors en présence de nourriture non pesée. 
Le lendemain matin, à l'heure choisie, nous enlevions les mangeoires à candi pour les remplacer par celles contenant du candi et du pollen, que nous avions préalablement pesées. L'expérience proprement dite commençait. Les abeilles étaient alors âgées de 1, 2, et quelquefois 3 jours.

Cette méthode permet à notre avis de diminuer le « stress » et d'homogénéiser les abeilles au sein des cagettes, notamment les accompagnatrices recevant une reine.

Nous avons toujours procédé de cette façon, sans jamais observer de perturbation dans le comportement. Notre protocole diffère en partie de celui de SrTbon (1967). Pour éviter, dans une certaine mesure, l'influence du « stress », cet auteur calcule la consommation journalière de ses abeilles en divisant par deux le chiffre global des 2 premiers jours d'alimentation. Il obtient ainsi une consommation moyenne identique pour les 2 premiers jours d'expérimentation. Or nous avons toujours observé, pour notre part, une différence notable entre ces deux premiers prélèvements. Nous avons pensé qu'il était intéressant de laisser apparaître cette différence.

Soulignons encore que les abeilles employées par SITBon sont âgées et prélevées à l'extérieur au mois de janvier. Il est donc raisonnable de penser que ces abeilles subissent un « stress» bien plus important que de jeunes abeilles fraîchement écloses en étuve.

$$
\text { 5. - Les pesées }
$$

Nous connaissions la tare de chaque mangeoire, la quantité de nourriture contenue dans chacune d'elle; les pesées quotidiennes nous donnaient par simple différence, la quantité de nourriture prélevée par les abeilles de chaque cagette.

Nous corrigions ce résultat en tenant compte :

- de la dessication que subissent les aliments placés en étuve à la température de $32^{\circ} \mathrm{C}$.

_ de la mortalité des abeilles.

\section{6. - Analyse statistique des résultats (tableau 1)}

Pour l'interprétation statistique des résultats, nous avons appliqué le test F de SNEDECoR déjà utilisé précédemment (Roger, PaIn, 1966). Il nous a paru intéressant d'analyser nos expériences sur 2 périodes. La première s'étend du 1 er au $7 \mathrm{e}$ jour. Elle correspond à la consommation réelle de candi et de pollen. La seconde période englobe les 15 jours de l'expérience. Elle comprend donc à la fois la période de consommation et les phases relatives à la construc. tion et au stockage de l'aliment sucré.

Le test porte sur l'ensemble des prélèvements ou des consommations enregistrés quotidiennement au sein de chaque lot de cagettes (témoins et traités). Les données sont corrigées en fonction de la mortalité des abeilles et de la dessication des aliments. Avant de procéder à l'analyse statistique, la prise de nourriture est rapportée à une abeille pour chaque lot considéré.

\section{RESULTATS}

Nous avons réalisé 7 séries d'expériences. Les reines employées, au nombre de 115, étaient des reines vierges ou fécondes, jeunes ou vieillies artificiellement en étuve.

Pour la commodité et la compréhension de l'exposé, nous analyserons nos expériences sans tenir compte de leur déroulement chronologique, mais uniquement en considérant la qualité des reines (vierges, fécondes, ...).

De même, nous parlerons de consommation pour les résultats obtenus avec l'aliment pollinique, et de prise de nourriture en ce qui concerne l'aliment sucré. Les abeilles prélèvent beaucoup plus de candi que de pollen, et ce dernier est complètement ingéré. Après 7 à 8 jours de claustration, les abeilles ont étiré le morceau de cire gaufrée mis à leur disposition. Elles ont façonné 
de véritables cellules et commencent à stocker le candi sous forme de sirop au fond des alvéoles. C'est surtout le cas des abeilles accompagnatrices de reine. Il n'y a donc pas une véritable consommation, mais un prélèvement de nourriture.

\section{1. - Expériences réalisées en présence de reines vierges.}

\section{Description des expériences.}

\section{Expérience $n^{0} 1$.}

Cette expérience a été effectuée en juillet 1966. Elle porte sur 2 lots de 15 cagettes. Chacune d'elle contient 50 ouvrières âgées de 1 et 2 jours, soit 750 abeilles par lot. Nous avons utilisé des reines vierges âgées d'une semaine.

Une abeille en présence de reine a prélevé en 15 jours $196 \mathrm{mg}$ de candi, et une abeille témoin $191,1 \mathrm{mg}$. Le prélèvement moyen dans les deux lots paraît sensiblement égal : $13,1 \mathrm{mg} / \mathrm{ab} / \mathrm{jour}$ et $12,7 \mathrm{mg} / \mathrm{ab} /$ jour. (fig. 1 ).

Après 7 jours, la consommation totale de pollen s'élève à $40,8 \mathrm{mg} / \mathrm{ab}$ pour les témoins, et $34,7 \mathrm{mg} / \mathrm{ab}$ pour les traitées, soit une ingestion moyenne respective de : 5,8 et $4,9 \mathrm{mg} / \mathrm{ab} /$ jour (fig. 2 ).

Nous obtenons une valeur de $F$ non significative pour les prélèvements de candi des deux périodes considérées.

La valeur de $F$ est négative aussi pour la consommation de pollen.

Expérience $n^{0} 2$.

En juin 1967, nous avons procédé à une seconde expérience avec de jeunes reines vierges, en nous rapprochant le plus possible des conditions expérimentales réunies au cours de la 1 ere expérience.

Elle porte sur 2 lots de 10 cagettes, contenant chacune 50 abeilles de 1 à 2 jours. Les reines vierges introduites auprès des petites populations d'abeilles claustrées, sont âgées cette fois de 18 jours. C'est la seule différence existant entre les expériences 1 et 2 .

En examinant la figure 3 , nous voyons que la courbe des prélèvements de candi effectués par les abeilles traitées, se maintient très nettement audessus de celle des témoins, du $1^{\text {er }}$ au dernier jour de l'expérience. Une abeille en présence de reine a prélevé en 15 jours $361,1 \mathrm{mg}$ de candi, et une abeille témoin $236,3 \mathrm{mg}$. Les moyennes atteignent respectivement dans les 2 lots : 24,1 et $15,7 \mathrm{mg} / \mathrm{ab} /$ jour (fig. 3 ).

La consommation totale de pollen d'une abeille orpheline (fig. 4) s'élève 


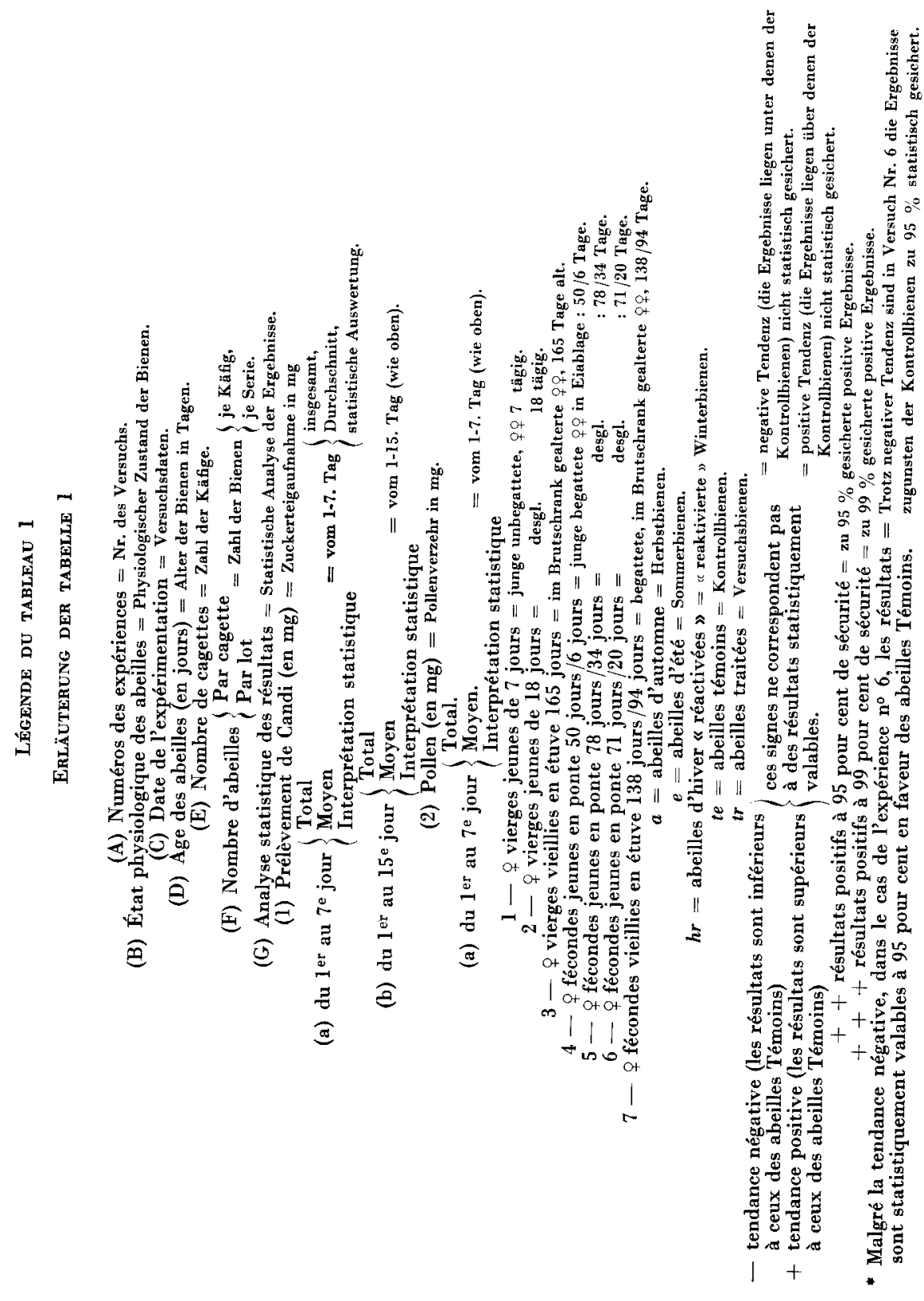




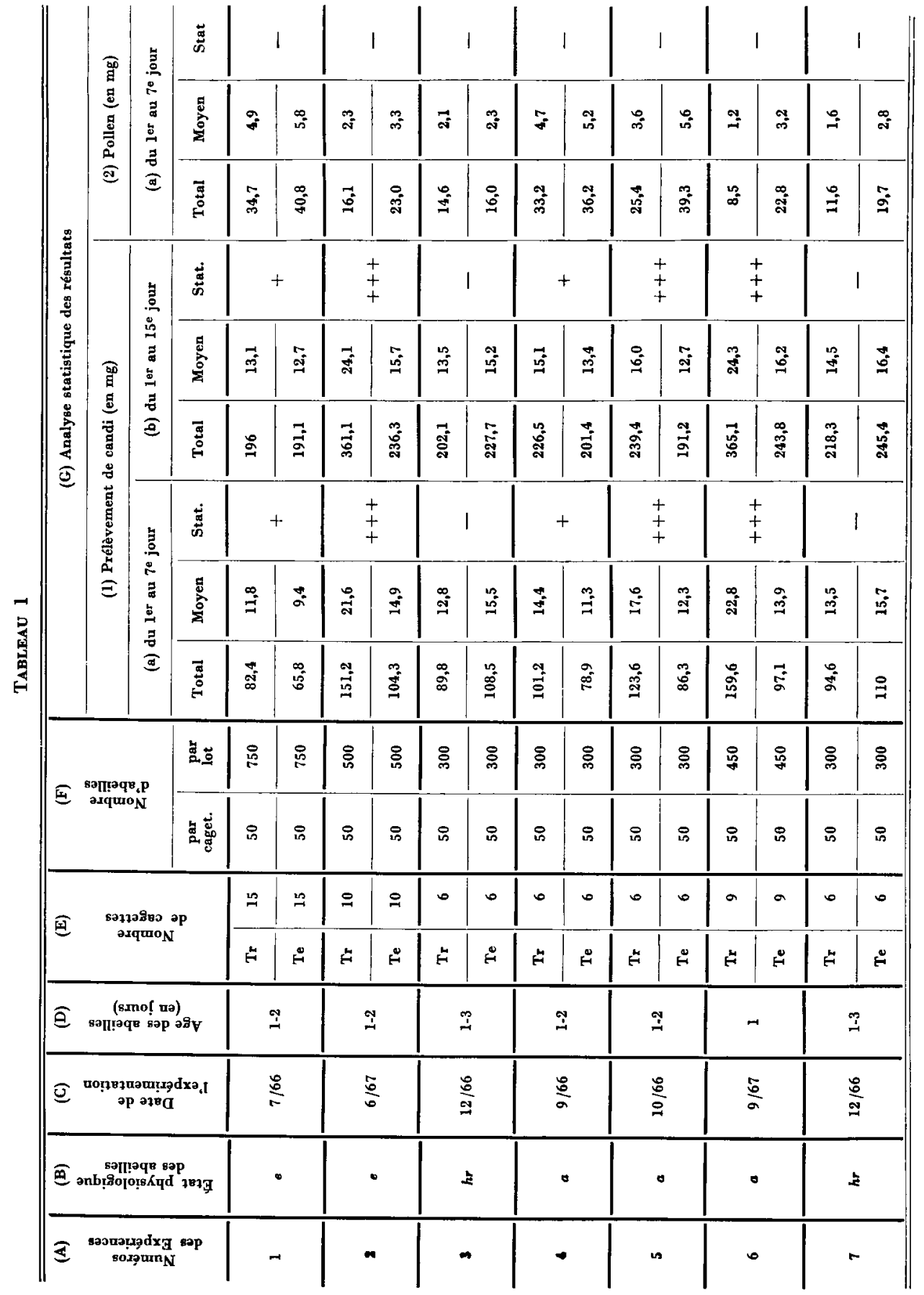


B. ROGER

à $23 \mathrm{mg}$ et $16,1 \mathrm{mg}$ pour une abeille traitée. Les moyennes respectives sont de 3,3 et $2,3 \mathrm{mg} / \mathrm{ab} / \mathrm{jour}$.

La valeur de $F$ est hautement significative en ce qui concerne la prise de nourriture sucrée, à la fois du $1^{\mathrm{er}}$ au $7^{\mathrm{e}}$ jour, et du $1^{\mathrm{er}}$ au $15^{\mathrm{e}}$ jour.

Le calcul indique pour $F$, les valeurs respectives de $: 10,79$ et 34,75 . Au seuil de $1 \%$, la table de sNedecor donne : 9,33 et 7,64.

L'analyse concernant le pollen donne un résultat non significatif.

Expériences $n^{\circ} 1$ et $n^{0} 2$ groupées - (fig. 5).

Lorsque nous groupons les résultats des expériences $n^{\text {os }} 1$ et 2 , nous obtenons pour les prélèvements de candi une valeur significative au seuil de $5 \%$ pour la première période. Le calcul indique pour $\mathrm{F}: 7,71$. Au seuil de $5 \%$ la table de SNEDECOR donne 4,26 et 7,82 au seuil de $1 \%$.

De même la valeur de $\mathrm{F}$ est significative pour l'ensemble de l'expérience. Le calcul indique pour $\mathrm{F}: 13,63$. Au seuil de $1 \%$ la table de snedecon donne 7,12 .

Pour le pollen, les expériences groupées fournissent des valeurs non significativement différentes.

\section{Expérience $n^{0} 3$.}

L'expérience $\mathrm{n}^{0} 3$ comprend 2 lots de 6 cagettes. Chacune d'elles contient 50 ouvrières âgées de 1,2 et 3 jours. Ces abeilles sont nées en décembre et proviennent de 3 cadres de couvain prélevés dans des ruches réactivées.

Les 6 reines utilisées font partie du même lot que celles dont nous nous sommes servis lors de la 1 ere expérience (juillet 1966). Mais nous les avons laissées vieillir en étuve pendant 165 jours avant d'entreprendre l'expérimentation.

En fin d'expérience, une ouvrière témoin a prélevé $227,7 \mathrm{mg}$, une abeille en présence de reine vierge vieillie, $202,1 \mathrm{mg}$ soit une moyenne de 15,2 et de $13,5 \mathrm{mg} / \mathrm{ab} /$ jour. (fig. 6).

Il est à remarquer que ce sont les abeilles témoins qui prélèvent le plus de nourriture sucrée.

La consommation de pollen s'élève à $16 \mathrm{mg} / \mathrm{ab}$ pour les témoins et $14,6 \mathrm{mg}$ / ab pour les traitées (fig. 7).

Les consommations moyennes sont de $: 2,3 \mathrm{mg} / \mathrm{ab}$ pour les témoins et $2,1 \mathrm{mg} / \mathrm{ab}$ pour les traitées.

La valeur $\mathrm{F}$ n'est significative, ni pour les deux périodes considérées ni pour les 2 sortes de nourriture. 
2. Comparaison des expériences réalisées avec reines vierges jeunes et reines vierges vieillies.

a) Importance de l'état de vieillissement des reines sur la prise de nourriture sucrée.

Dans la description de l'expérience $n^{0} 1$, nous avons signalé que les reines utilisées étaient âgées d'une semaine. Or, si les ouvrières qui les accompagnent prélèvent plus de candi que les témoins, la différence entre les deux lots étudiés est malgré tout assez faible. Elle est de $16,6 \mathrm{mg} / \mathrm{ab}$, après 7 jours et $4,9 \mathrm{mg} / \mathrm{ab}$ après 15 jours d'expérience.

Par contre, au cours de la seconde expérience, la différence de prise de nourriture s'avère nettement plus importante entre lot traité et lot témoin : $46,9 \mathrm{mg} / \mathrm{ab}$ après le $7 \mathrm{e}$ jour et $124,8 \mathrm{mg} / \mathrm{ab}$ au $15 \mathrm{e}$ jour de l'expérience. Dans ce cas les reines étaient âgées de 18 jours au début de l'expérimentation. Les résultats obtenus nous permettent de penser que ces reines influencent plus la prise de nourriture sucrée des abeilles encagées avec elles que les précédentes. Nous en discuterons plus loin.

Cependant lorsque les reines vierges sont âgées (165 jours de claustration, avec 6 changements de population) ce sont les abeilles orphelines qui prélèvent le plus de candi : $18,7 \mathrm{mg} / \mathrm{ab}$ après 7 jours et $25,6 \mathrm{mg} / \mathrm{ab}$ à la fin de l'expérience.

b) Importance de l'état de vieillissement des reines sur la consommation de pollen.

Bien que l'analyse statistique ne permette pas de conclusion formelle, on constate que les résultats convergent pour les 3 expériences : les abeilles privées de reine consomment un peu plus de pollen.

Nous pouvons noter aussi que la quantité de pollen prélevée au cours des différentes expériences, varie considérablement d'une expérience à l'autre et cela indépendamment du fait de la présence ou de l'absence de la reine. D'une manière générale les abeilles provenant de ruches situées à l'extérieur et prélevées à la belle saison (exp. 1 et 2 ) ont consommé davantage de pollen que celles issues de ruches réactivées (exp. 3). Ces dernières ont pourtant été conservées en étuve dans les mêmes conditions que les abeilles des expériences précédentes. Peut-être est-ce dû à un moins bon état physiologique.

La consommation totale par les abeilles des groupes témoins dans l'expérience $n^{\circ} 1$ et dans l'expérience $n^{\circ} 2$, est évaluée respectivement à : 40,8 mg et $23 \mathrm{mg}$.

Il est intéressant aussi d'observer les consommations moyennes. En effet, la différence entre les témoins et les traitées de l'expérience $n^{0} 1$ s'élève à : $0,9 \mathrm{mg} / \mathrm{ab} /$ jour $(5,8-4,9)$. Pour l'expérience $\mathrm{n}^{0} 2$, nous obtenons : $0,9 \mathrm{mg} / \mathrm{ab} /$ jour $(3,2-2,3)$, soit le même chiffre. 
Autrement dit, bien que les quantités totales de pollen prélevées soient fort différentes dans les 2 expériences que nous comparons, une abeille témoin consomme en moyenne, dans les deux cas, $0,9 \mathrm{mg}$ par jour de plus qu'une abeille traitée. L'écart entre les moyennes considérées est identique. Quant aux abeilles mises en présence d'une reine vierge vieillie, leur consommation est pratiquement identique à celle des abeilles témoins (2,1 et $2,3 \mathrm{mg} / \mathrm{ab} /$ jour). Notons toutefois que les prélèvements de pollen les plus faibles sont enregistrés au cours de cette expérience.

\section{Conclusion partielle.}

Les trois expériences que nous venons de décrire nous ont permis de mettre en évidence les points suivants :

- les ouvrières en présence de reines vierges jeunes prélèvent plus de matière sucrée que les abeilles orphelines;

- au contraire, les abeilles claustrées avec des reines vierges vieillies prélèvent moins de candi que les abeilles témoins;

- ce sont toujours les abeilles orphelines qui consomment le plus de pollen.

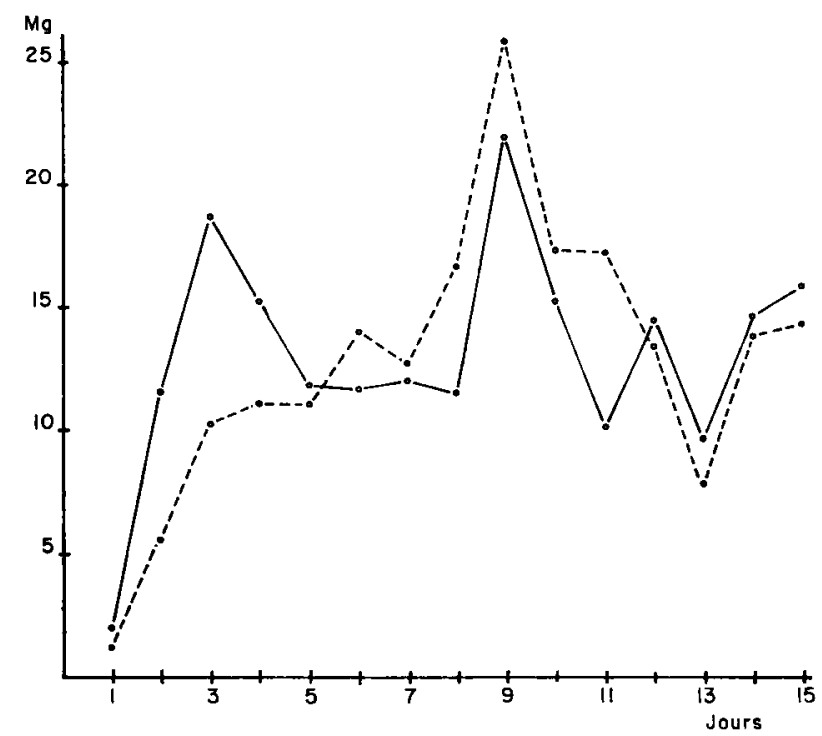

Fig. 1. - Expérience n ${ }^{\circ}$ 1. Reines vierges de 7 jours.

— Prélèvement de candi par une abeille traitée (en présence de reine).

.... Prélèvement de candi par une abeille témoin (orpheline).

Aвв. 1. - Versuch Nr. 1. Unbegattete, 7 tägige 우.

Zuckerteigaufnahme einer Versuchsbiene in Anwesenheit einer .

..... Zuckerteigaufnahme einer weisellosen Kontrollbiene. 


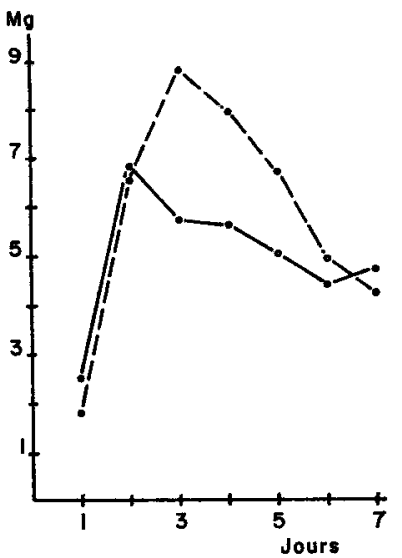

Frg. 2. - Expêrience no 1.

Prélèvement de pollen par une abeille traitée (en présence de reine). Prélèvement de pollen par une abeille témoin (orpheline).

Авв. 2. - Versuch $\mathrm{Nr} .1$.

Pollenverzehr einer Versuchsbiene in Anwesenheit einer Königin.

Pollenverzehr einer weisellosen Kontrollbiene.

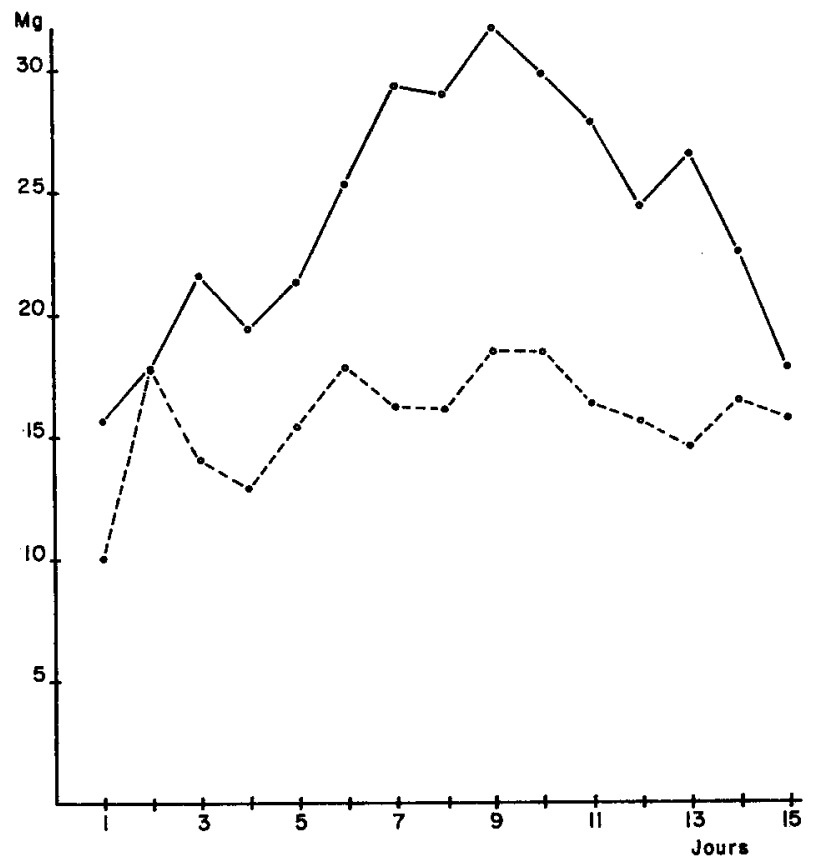

FIG. 3. - Expérience $n^{\circ}$ 2. Reines vierges de 18 jours.

Prélèvement de candi par une abeille traitée (en présence de reine). Prélèvement de candi par une abeille témoin (orpheline).

Aвн. 3. - Versuch Nr. 2. Unbegattete, 18 tägige 우․

Zuckerteigaufnahme einer Versuchsbiene in Anwesenheit einer 9. Znckerteigaufnahme einer weisellosen Kontrollbiene. 


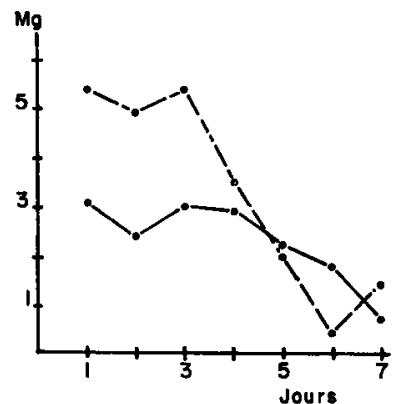

Frc. 4. - Expérience n० 2.

— Prélèvement de pollen par une abeille traitée (en présence de reine). .... Prélèvement de pollen par une abeille témoin (orpheline).

Aв8. 4. - Versuch Nr. 2.

- Pollenverzehr einer Versuchsbiene in Anwesenheit einer Königin. ..... Pollenverzehr einer weisellosen Kontrollbiene.

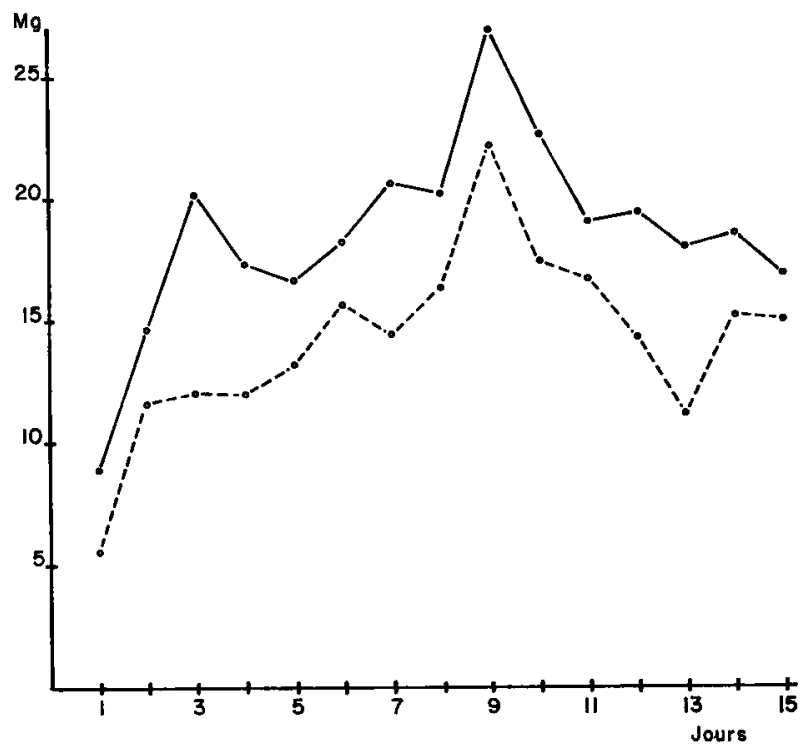

Fıc. 5. - Expériences $n^{08} 1$ et 2 groupees. Reines vierges jeunes.

Prélèvement de candi par une abeille traitée (en présence de reine). .... Prélèvement de candi par une abeille témoin (orpheline).

ABB. 5. - Versuch Nr. 1 u. 2 zusammengefasst; junge unbegattete

- Zuckerteigaufnahme einer Versuchsbiene in Anwesenheit einer 9 . ..... Zuckerteigaufnahme einer weisellosen Kontrollbiene. 


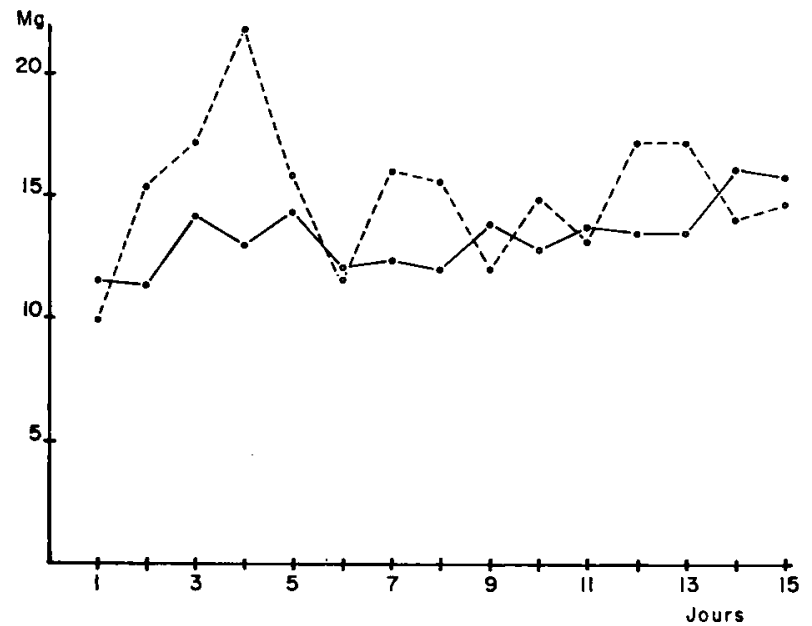

FIg. 6. - Expérience n⿳0 3. Reines vierges de 165 jours.

Prélèvement de candi par une abeille traitée (en présence de reine). Prélèvement de candi par une abeille témoin (orpheline).

Авв. 6. - Versuch Nr. 3 Unbegattete

Zuckerteigaufnahme einer Versuchsbiene in Anwesenheit einer $q$.

.... Zuckerteigaufnahme einer weisellosen Kontrollbiene.

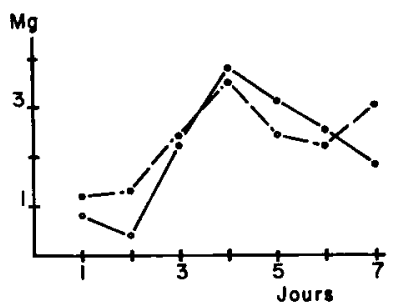

Fig. 7. - Expérience no 3.

- Prélèvement de pollen par une abeille traitée (en présence de reine).

....- Prélèvement de pollen par une abeille témoin (orpheline).

Авв. 7. - Versuch $\mathrm{Nr} .3$.

- Pollenverzehr einer Versuchsbiene in Anwesenheit einer Königin.

.... . Pollenverzehr einer weisellosen Krontrollbiene.

2. - Expériences réalisées en présence de reines fécondes.

\section{Description des expériences.}

Expérience $n^{0} 4$.

Cette expérience a été effectuée en septembre 1966. Elle porte sur 2 lots de 6 cagettes contenant chacune 50 abeilles de 1 à 5 jours, soit 300 abeilles par lot. 
Les reines utilisées sont de jeunes reines fécondes âgées de 50 jours, dont 44 jours de vie en ruchettes 3 cadres, situées à l'extérieur, et 6 jours de vie en cagette dans une étuve à la température de $32^{\circ} \mathrm{C}$.

Comme pour toutes les autres expériences effectuées avec des reines fécondes, nous avons exercé plusieurs contrôles portant sur la ponte et sur l'état et l'importance du couvain produit par les reines destinées aux expériences.

En fin d'expérience, le prélèvement global d'une abeille témoin s'élève à $201,4 \mathrm{mg}$, celui d'une abeille traitée à $226,5 \mathrm{mg}$.

Nous obtenons les prélèvements moyens suivants : $13,4 \mathrm{mg} / \mathrm{ab}$ chez les témoins et $15,1 \mathrm{mg} / \mathrm{ab}$ chez les traitées (fig. 8).

Les quantités totales de pollen ingéré par les abeilles des deux lots sont assez proches l'une de l'autre : 36,2 et $33,2 \mathrm{mg} / \mathrm{ab}$.

Les consommations moyennes journalières s'établissent ainsi : 5,2 mg/ab pour une ouvrière témoin, et $4,7 \mathrm{mg} / \mathrm{ab}$ pour une ouvrière traitée (fig. 9).

Nous obtenons une valeur de $F$ non significative pour les deux périodes concernant les prélèvements de candi. La valeur de $F$ est non significative pour la consommation du pollen.

\section{Expérience no 5.}

Cette expérience a été effectuée au début du mois d'octobre 1966, avec des reines issues du même lot que celles utilisées lors de l'expérience précédente $\left(\exp . n^{\circ} 4\right)$.

Au moment de l'expérience ces reines sont en pleine ponte et un peu plus âgées. Elles ont 78 jours de vie dont 44 jours passés en ruchettes 3 cadres et 34 jours en cagette dans une étuve à $32^{\circ} \mathrm{C}$, ce qui représente un vieillissement supplémentaire de 28 jours, effectué en cagette.

Les abeilles accompagnatrices ont 1 et 2 jours de vie. Chaque lot comprend 6 cagettes de 50 ouvrières.

La courbe de prélèvement des abeilles en présence de reine se maintient au-dessus de celle des abeilles témoins 14 jours sur 15 , mis à part le $8^{\mathrm{e}}$ jour, où nous obtenons le plus faible prélèvement effectué par les abeilles traitées, $11,7 \mathrm{mg} / \mathrm{ab}$.

A la fin de l'expérience, les prélèvements totaux atteignent $191,2 \mathrm{mg} / \mathrm{ab}$ pour les ouvrières témoins, et $239,4 \mathrm{mg} / \mathrm{ab}$ pour les ouvrières traitées, soit une prise de nourriture moyenne respective de 12,7 et $16 \mathrm{mg} / \mathrm{ab}$. (fig. 10).

Pour le pollen nous obtenons les consommations totales suivantes : $39,3 \mathrm{mg} / \mathrm{ab}$ pour les ouvrières témoins et $25,4 \mathrm{mg} / \mathrm{ab}$ pour les traitées.

Les moyennes respectives s'élèvent à 5,6 et $3,6 \mathrm{mg} / \mathrm{ab} / \mathrm{jour}$. (fig. 11).

La valeur de $F$ est hautement significative pour la prise de nourriture sucrée, à la fois sur 7 et sur 15 jours.

Le calcul indique pour $F$ les valeurs respectives suivantes : 30,13 et 14,37 . Au seuil de $1 \%$ la table de sNEDECOR donne 9,33 et 7,64. 
La valeur de $F$ est non significative pour la consommation de pollen.

\section{Expérience no 6.}

Cette expérience s'est déroulée en septembre 1967. Nous avons utilisé 2 lots de 9 cagettes contenant chacune 50 ouvrières de 1 jour.

Au commencement de l'observation, les reines fécondes sont âgées de 71 jours : 51 jours en ruchettes 3 cadres, et 20 jours en cagette. Elles sont en pleine ponte. Les expériences 5 et 6 sont donc comparables.

Du $1^{\text {er }}$ au $15^{\mathrm{e}}$ jour de cette expérience, la courbe des abeilles traitées se maintient nettement au-dessus de celle des abeilles témoins. A la fin de l'expérience, c'est-à-dire au $15^{\mathrm{e}}$ jour, une abeille traitée a prélevé $365,1 \mathrm{mg}$ et une abeille témoin $243,8 \mathrm{mg}$, soit un prélèvement moyen respectif de : 24,3 et $16,2 \mathrm{mg} / \mathrm{ab} /$ jour (fig. 12).

La courbe de consommation des abeilles orphelines se maintient tout au long de l'expérience au-dessus de celle des abeilles en présence de reine.

Pour le pollen nous obtenons les consommations totales suivantes : $22,8 \mathrm{mg} / \mathrm{ab}$ chez les témoins, contre seulement $8,5 \mathrm{mg} / \mathrm{ab}$ chez les traitées.

Les moyennes quotidiennes s'élèvent respectivement à 3,2 et $1,2 \mathrm{mg} / \mathrm{ab}$ (fig. 13).

La valeur de $F$ est hautement significative pour les prélèvements de candi au cours des deux périodes. Le calcul indique pour $\mathrm{F}$ les valeurs respectives suivantes : 16, 78 et 43, 17. Au seuil de $1 \%$ la table de sNeDEcor donne 9,33 et 7,64 .

Pour la consommation de pollen, la valeur de $F$ est significative au seuil de $5 \%$. Le calcul indique pour F 5,50. La table de sneDEcor donne 4,20 à $5 \%$ et 7,64 à $1 \%$.

\section{Expériences nos 4,5 et 6 groupées (fig. n 14).}

Lorsque nous examinons ensemble les expériences 4, 5 et 6 ou bien lorsque nous les groupons deux à deux $(4+5,5+6,4+6)$ la valeur de $F$ est hautement significative pour les deux périodes considérées.

Par contre, la valeur de $\mathbf{F}$ reste non significative lorsque nous groupons de la même façon les différents résultats concernant la consommation de pollen.

\section{Expérience $n^{\circ} 7$.}

Nous avons disposé pour cette expérience de 600 abeilles réparties en 2 lots de 6 cagettes. Chacune de ces cagettes contenait 50 abeilles de 1, 2 et 3 jours. Ce sont des abeilles provenant de couvain prélevé dans des ruches réactivées.

Nous avons utilisé des reines âgées de 138 jours au début de l'expéri- 
mentation. Ces reines étaient en pleine ponte. Elles avaient 44 jours de vie en ruchettes 3 cadres et 94 jours de vie en cagette dans une étuve à $32^{\circ} \mathrm{C}$.

La quantité totale de nourriture prélevée se monte à $245,4 \mathrm{mg} / \mathrm{ab}$ pour une ouvrière témoin et à $218,3 \mathrm{mg} / \mathrm{ab}$ pour une ouvrière traitée, soit les moyennes respectives de 16,4 et $14,5 \mathrm{mg} / \mathrm{ab}$ (fig. $\mathrm{n}^{\circ} 15$ ).

Les consommations totales de pollen s'élèvent à $19,7 \mathrm{mg} / \mathrm{ab}$ chez les témoins et $11,6 \mathrm{mg} / \mathrm{ab}$ chez les traitées. Nous obtenons dans le même ordre, les moyennes journalières suivantes : 2,8 et $1,6 \mathrm{mg} / \mathrm{ab}$.

Une abeille orpheline consomme donc presque 2 fois plus de pollen qu'une abeille en présence de reine (fig. 16).

La valeur de $F$ est non significative à la fois pour les deux périodes considérées et pour les deux sortes de nourriture.

2. Comparaison des expériences réalisées avec reines fécondes jeunes et reines fécondes vieillies.

a) Importance de l'état de vieillissement des reines sur la prise de nourriture sucrée.

L'expérience $n^{\circ} 4$ commence alors que les reines sont âgées de 50 jours, dont 44 jours passés en ruchettes 3 cadres. Les six reines, dont nous avons contrôlé le couvain sont en pleine ponte. Sur l'ensemble de l'expérience, les abeilles en présence de reine ont consommé davantage de candi que les abeilles témoins. Après les 7 premiers jours, l'écart moyen entre les 2 lots s'élève à $22,3 \mathrm{mg} / \mathrm{ab}$ en faveur des ouvrières traitées. Cette différence passe à $25,1 \mathrm{mg} / \mathbf{a b}$ en fin d'expérimentation.

Pour les expériences $\mathrm{n}^{\text {os }} 5$ et 6 , les reines utilisées sont assez comparables : 78 et 71 jours de vie au moment de leur utilisation, dont au minimum 44 jours passés en ruchettes 3 cadres (exp. no 5 ). Les abeilles en présence de reines prélèvent davantage de nourriture sucrée.

Au cours de l'expérience no 5 , nous obtenons les écarts moyens suivants : $37,3 \mathrm{mg} / \mathrm{ab}$ après le $7^{\mathrm{e}}$ jour d'expérience et $48,2 \mathrm{mg} / \mathrm{ab}$ en fin d'expérience.

Si nous examinons maintenant les résultats obtenus pour l'expérience $n^{0}$ 6, nous notons qu'ils vont dans le même sens. En effet, les abeilles traitées prélèvent davantage de candi que les témoins. Mais les écarts entre les deux lots d'abeilles observées sont plus importants que ceux enregistrés au cours de l'expérience $\mathbf{n}^{\circ} 5$.

Les différences atteignent $62,5 \mathrm{mg} / \mathrm{ab}$ après le $7^{\mathrm{e}}$ jour et $121,3 \mathrm{mg} / \mathrm{ab}$ après le 15 e jour.

L'expérience $n^{\circ} 7$ comprend comme nous l'avons vu, des reines âgées de 138 jours, dont 44 jours de vie en ruchette 3 cadres, et 94 jours de vie en cagette dans une étuve à $32^{\circ} \mathrm{C}$. $\mathrm{Or}$, ce sont cette fois les abeilles orphelines 
qui prélèvent le plus de candi; après le $7^{\mathrm{e}}$ jour, l'écart entre les deux lots atteint 15,4 mg/ab. En fin d'expérience, cet écart passe à 27,1 mg/ab. Pour les expériences $\mathrm{n}^{\mathrm{OS}} 4,5$ et 6 , les différences de prélèvement de candi entre lots témoins et lots traités semblent proportionnelles au vieillissement des reines et à l'importance du temps passé par celles-ci à l'intérieur des cagettes.

Au cours de l'expérience $n^{\circ} 4$ les reines fécondes ont séjourné seulement 6 jours en cagette et dans ce cas nous obtenons une prise de nourriture légèrement supérieure en faveur des abeilles traitées, mais un résultat statistique non significatif. Ces conclusions sont comparables à celles de l'expérience $n^{0} 1$ avec des reines vierges âgées d'une semaine.

Par contre, pour les expériences 4,5 et 2, le séjour des reines en cagette a duré respectivement 34,20 et 18 jours. Or nous obtenons pour ces trois expériences des valeurs statistiquement significatives. Les ouvrières accompagnatrices de reines prélèvent plus de nourriture sucrée que les abeilles orphelines (voir tableau no 1 ).

b) Importance de l'état de vieillissement des reines sur la prise de nourriture azotée.

Si nous considérons les expériences $n^{\text {os }} 4,5,6$ et 7 nous observons des résultats analogues. Dans tous les cas les abeilles témoins, orphelines, consomment plus de pollen que les abeilles claustrées en présence de reine. Toutefois, les abeilles provenant de ruches réactivées semblent manger moins de pollen.

\section{Conclusion partielle.}

Les résultats obtenus pour les 4 expériences réalisées avec des reines fécondes, nous permettent de souligner les points suivants :

- les prélèvements de candi effectués par les abeilles en présence de jeunes reines fécondes sont nettement plus élevés que ceux réalisés par les abeilles orphelines (voir fig. 14 - exp. groupées);

- par contre les abeilles accompagnatrices de reines fécondes vieillies prélèvent moins de nourriture sucrée que les abeilles témoins ;

- ce sont toujours les abeilles orphelines qui consomment le plus de pollen. 


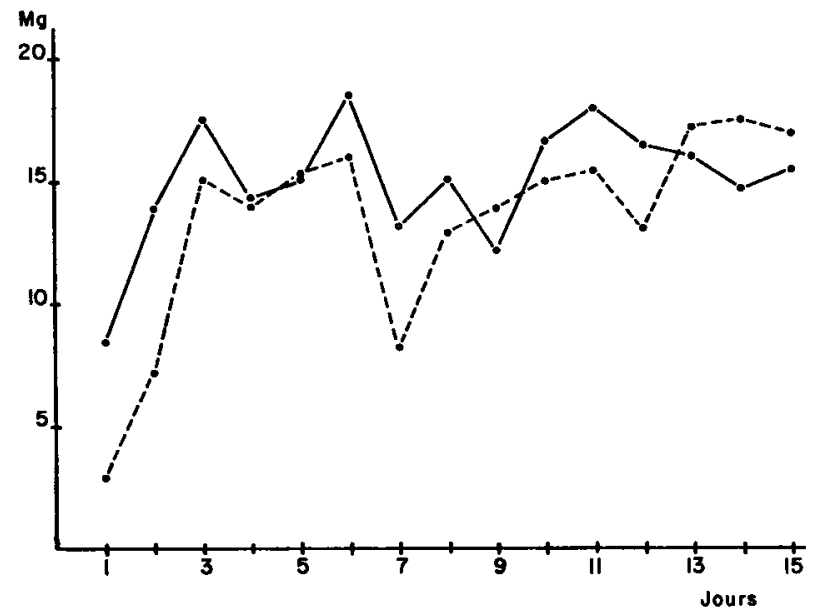

Fic. 8. - Expérience no 4. Reines fécondes de 50 jours.

Prélèvement de candi par une abeille traitée (en présence de reine).

..... Prélèvement de candi par une abeille témoin (orpheline).

Aвв. 8. - Versuch Nr. 4. Begattete $\nmid \circ, 50$ Tage alt.

Zuckerteigaufnahme einer Versuchsbiene in Anwesenheit einer .

..... Zuckerteigaufnahme einer weisellosen Kontrollbiene.

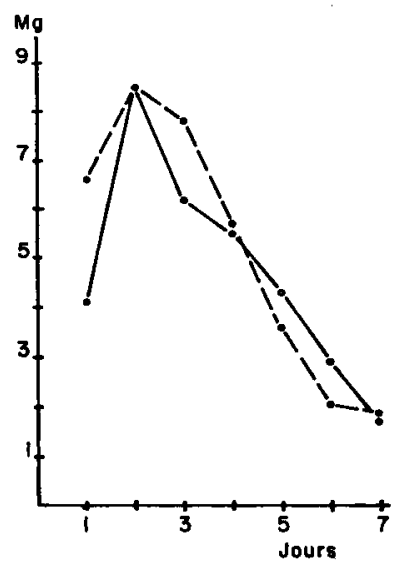

Fig. 9. - Expérience no 4.

Prélèvement de pollen par une abeille traitée (en présence de reine). Prélèvement de pollen par une abeille témoin (orpheline).

Aв8. 9. - Versuch Nr. 4.

Pollenverzehr einer Versuchsbiene in Anwesenheit einer Königin. Pollenverzehr einer weisellosen Kontrollbiene. 


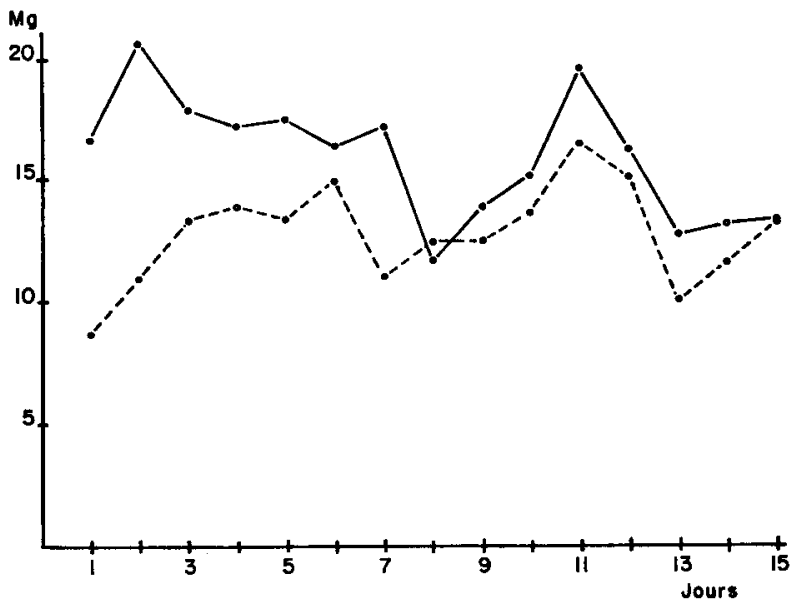

Fic. 10. - Expérience no 5 . Reines fécondes de 78 jours.

- Prélèvement de candi par une abeille traitée (en présence de reine). Prélèvement de candi par une abeille témoin (orpheline).

Aв8. 10. - Versuch Nr. 5. Begattete 우, 78 Tage alt.

Zuckerteigaufnahme einer Versuchsbiene in Anwesenheit einer o.

_.... Zuckerteigaufnahme einer Versuchsbiene in Anwesenhe

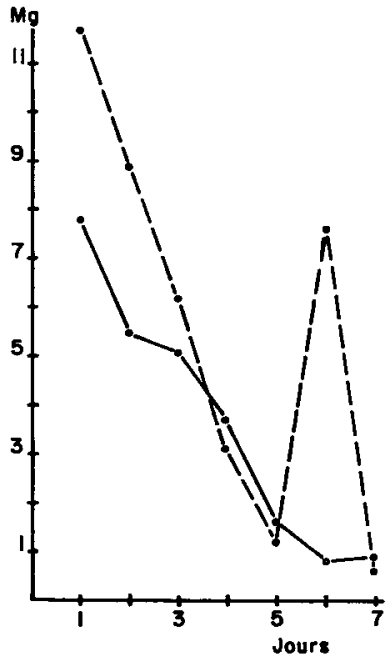

FIG. 11. - Expérience no 5 .

Prélèvement de pollen par une abeille traitée (en présence de reine). Prélèvement de pollen par une abeille témoin (orpheline).

Авв. 11. - Versuch Nr. 5.

Pollenverzehr einer Versuchsbiene in Anwesenheit einer Königin. Pollenverzehr einer weisellosen Kontrollbiene. 


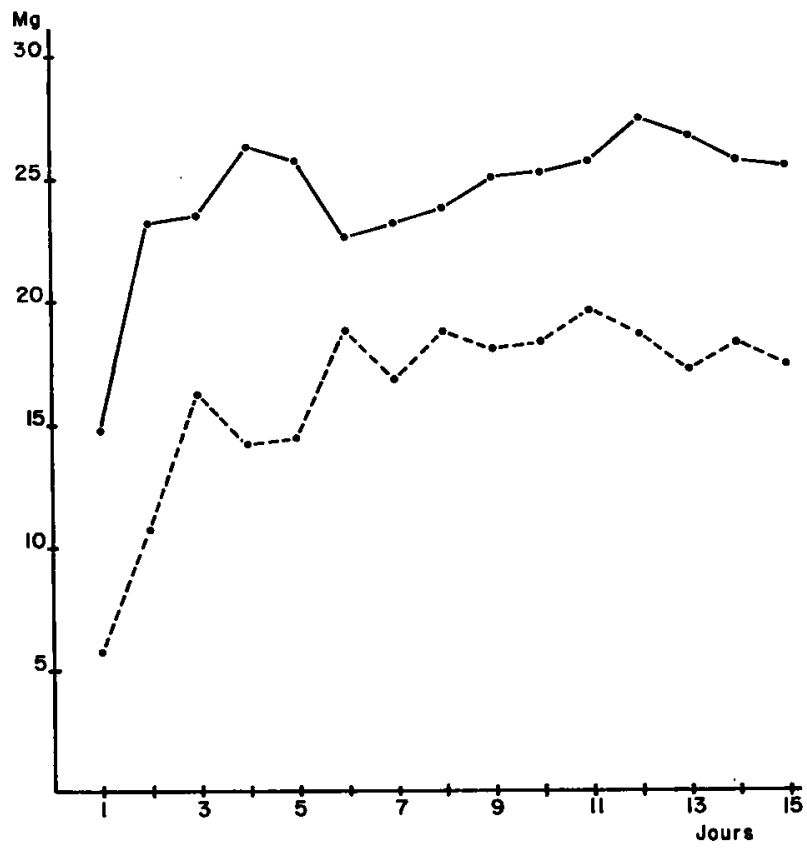

Fig. 12. - Expérience n' 6. Reines fécondes de 71 jours.

Prélèvement de candi par une abeille traitée (en présence de reine).

..... Prélèvement de candi par une abeille témoin (orpheline).

Aв8. 12, - Versuch Nr. 6. Begattete 우, 71 Tage alt.

- Zuckerteigaufnahme einer Versuchsbiene in Anwesenheit einer 우.

..... Zuckerteigaufnahme einer weisellosen Kontrollbiene.

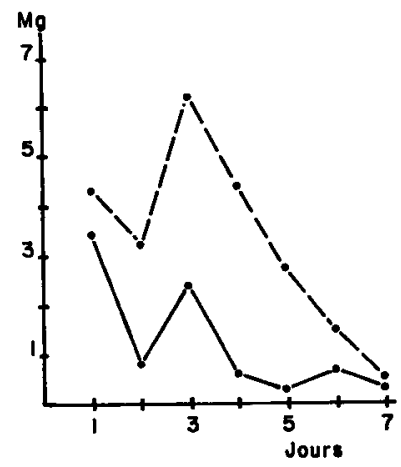

Fig. 13. - Expérience $n^{\circ} 6$.

Prélèvement de pollen par une abeille traitée (en présence de reine). Prélèvement de pollen par une abeille témoin (orpheline).

Aв8. 13. - Versuch Nr. 6.

Pollenverzehr einer Versuchsbiene in Anwesenheit einer Königin.

..... Pollenverzehr einer weisellosen Kontrollbiene. 


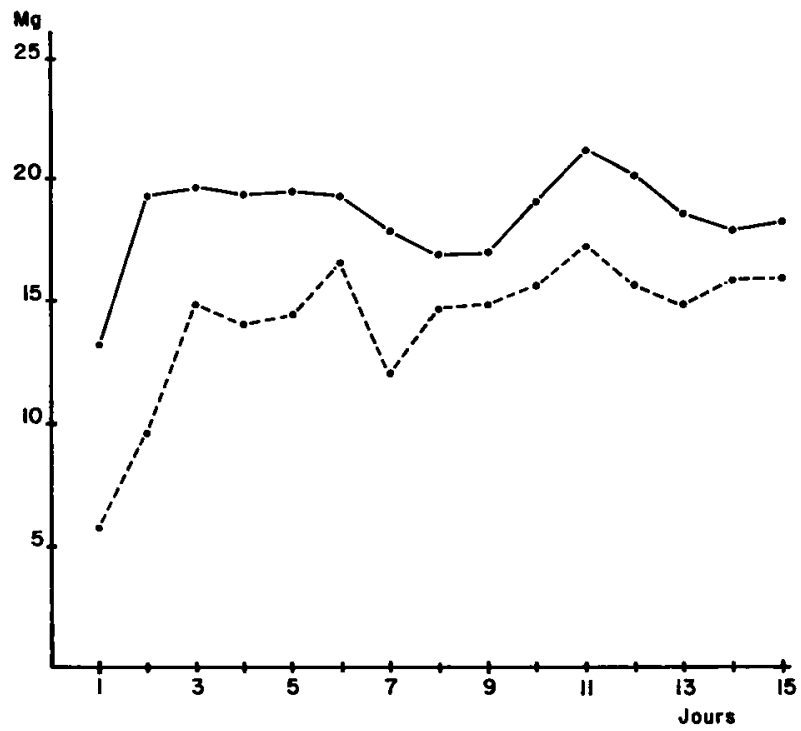

Fig. 14. - Expériences $n^{o s} 4,5$ et 6 groupées. Reines fécondes jeunes.

Prélèvement de candi par une abeille traitée (en présence de reine). Prélèvement de candi par une abeille témoin (orpheline).

AвB. 14. - Versuche Nr.4, 5 und 6 zusammengefasst. Junge begattete 우.

Zuckerteigaufnahme einer Versuchsbiene in Anwesenheit einer 우.

..... Zuckerteigaufnahme einer weisellosen Kontrollbiene.

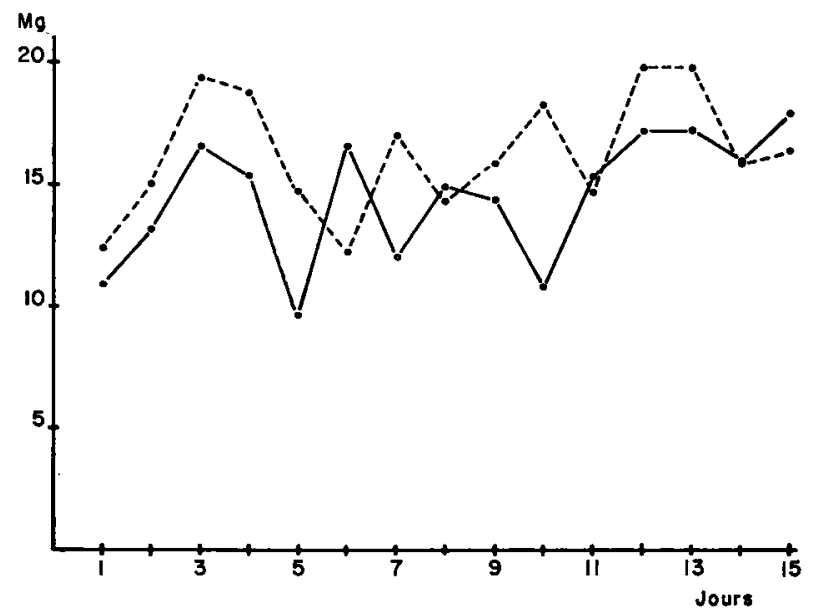

Fic. 15. - Expérience $n^{\circ}$ 7. Reines fécondes de 138 jours.

- Prélèvement de candi par une abeille traitée (en présence de reine). .... - Prélèvement de candi par une abeille témoin (orpheline).

Aвв. 15. - Versuch Nr. 7. Begattete $9{ }^{\circ}, 138$ Tage alt.

Zuckerteigaufnahme einer Versuchsbiene in Anwesenheit einer $q$.

..... Zuckerteigaufnahme einer weisellosen Kontrollbiene. 


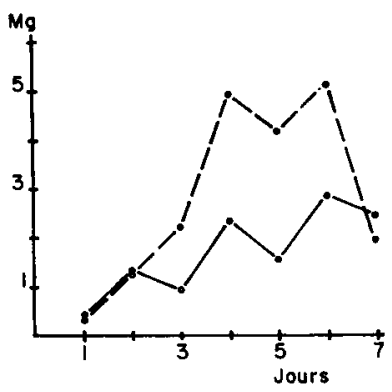

FIG. 16. - Expérience $n^{\circ} 7$.

- Prélèvement de pollen par une abeille traitée (en présence de reine).

..... Prélèvement de pollen par une abeille témoin (orpheline).

Авв. 16. -.- Versuch $\mathrm{Nr} .7$.

Pollenverzehr einer Versuchsbiene in Anwesenheit einer Königin.
Pollenverzehr einer weisellosen Kontrollbiene.

\section{3. - Interprétation des courbes}

1. Observations relatives au prélèvement de candi.

Nous avons vu que les prélèvements de candi sont toujours plus importants pour des populations recevant une jeune reine, vierge ou féconde.

Chez les abeilles accompagnatrices de jeunes reines vierges (Exp. ${ }^{\text {os }} 1$ et 2 ), nous obtenons dans les deux cas un premier pic, dès le $3^{\mathrm{e}}$ jour. Le lendemain les prélèvements diminuent de façon sensible, surtout pour l'expérience $\mathbf{n}^{0} 1$. Ils deviennent ensuite constants pour cette dernière expérience, mais par contre de plus en plus importants pour l'expérience $n^{\circ} 2$. Nous enregistrons un second pic (prélèvement maximum dans les 2 cas) au cours du 9 e jour. Ensuite la prise de nourriture diminue dans l'expérience $\mathbf{n}^{\circ} 2$, presque régulièrement, alors qu'elle subit des variations dans l'expérience $n^{0} 1$.

Les prélèvements effectués le $1^{\mathrm{er}}$ jour par les abeilles accompagnatrices de jeunes reines fécondes, sont relativement importants par rapport aux témoins. Dans l'expérience $n^{\circ} 4$ nous avons un $1^{\text {er }}$ pic le $3^{\mathrm{e}}$ jour, pour l'expérience $\mathrm{n}^{\mathrm{O}} 5$ il se situe au $2^{\mathrm{e}}$ jour et pour l'expérience $\mathrm{n}^{\mathrm{O}} 6$ nous obtenons 2 pics, le $2^{\mathrm{e}}$ et surtout le $4^{e}$ jour. Ensuite les prises de nourriture varient sensiblement d'une expérience à l'autre. Enfin au $11^{\mathrm{e}}$ jour nous trouvons de nouveau un pic important pour les expériences $n^{\text {os }} 4$ et 5 et au $12^{\mathrm{e}}$ jour pour l'expérience $n^{0} 6$.

Si nous comparons entre elles les expériences avec reines vierges et reines fécondes jeunes, nous constatons que :

- Le premier pic se situe pour toutes les expériences entre le $2^{\mathrm{e}}$ et le $4^{\mathrm{e}}$ jour. Les abeilles ont alors le plus souvent le même âge, soit entre 3 et 4 jours.

Le second pic important apparaît au $9 \mathrm{e}$ jour chez les reines vierges jeunes, et au $11^{\mathrm{e}}$ et $12^{\mathrm{e}}$ jour pour les reines fécondes jeunes, soit respectivement entre 
le $9^{\mathrm{e}}$ et le $10^{\mathrm{e}}$ jour de vie, et le $11^{\mathrm{e}}$ et $13^{\mathrm{e}}$ jour de vie des ouvrières accompagnatrices.

- Chez les abeilles en présence de reines vieillies (Exp. nos 3 et 7) le $1^{\text {er }}$ pic est enregistré le $3^{\mathrm{e}}$ jour, les abeilles étant âgées de 3 à 6 jours. Puis après des variations plus ou moins prononcées selon l'expérience considérée, nous notons un accroissement de la prise de nourriture au cours des derniers jours : au 14e jour pour l'expérience $\mathbf{n}^{0} 3$ et au $15^{\mathrm{e}}$ jour pour l'expérience $\mathbf{n}^{0} 7$.

Chez les abeilles témoins sur l'ensemble des 7 expériences, le 1 er jour se traduit par une faible prise de nourriture sucrée. Ensuite, sauf pour l'expérience $n^{\circ} 2$ où nous observons un pic important dès le $2^{\mathrm{e}}$ jour, les prélèvements s'accentuent pour atteindre un premier pic important au cours du $6^{\mathrm{e}}$ jour d'expérimentation, les abeilles étant âgées de 6 à 7 jours. Il est à noter que les abeilles d'été (Exp. $\mathbf{n}^{\text {os }} 1$ et 2) effectuent un second prélèvement intense au cours du $9^{\mathrm{e}}$ jour. Les abeilles d'automne réalisent ce second prélèvement important un peu plus tard, surtout le $11^{\mathrm{e}}$ jour. Enfin pour les abeilles provenant de ruches réactivées il a lieu le $10^{\mathrm{e}}, 12^{\mathrm{e}}$ et $13^{\mathrm{e}}$ jour.

Nous pouvons donc dire que les abeilles en présence de reines prélèvent plus vite des quantités élevées de nourriture sucrée, dès le $3^{\mathrm{e}}$ jour, et seulement à partir du $6^{\mathrm{e}}$ jour chez les témoins. Par la suite le rythme de la prise de nourriture semble devenir comparable dans les deux groupes, tout en restant presque toujours quantitativement supérieur chez les traitées.

\section{Observations relatives à la consommation de pollen.}

Les résultats obtenus pour l'ensemble des expériences vont tous dans le même sens, la consommation de pollen est plus importante chez les abeilles témoins.

Pour les abeilles claustrées en présence de jeunes reines vierges, la consommation maximum se situe respectivement le $2^{\mathrm{e}}$ jour, et les $1^{\mathrm{er}}$ et $3^{\mathrm{e}}$ jours.

Avec les jeunes reines fécondes les prises de nourriture les plus élevées ont lieu le $1^{\mathrm{er}}$ jour dans les expériences $\mathrm{n}^{\mathrm{os}} 5$ et 6 , et le $2^{\mathrm{e}}$ jour pour l'expérience $n^{0} 4$.

Enfin les abeilles accompagnatrices de reines vieillies, les pics apparaissent au $4^{\mathrm{e}}$ jour pour l'expérience $n^{0} 3$, et au $6^{\mathrm{e}}$ jour pour l'expérience $n^{0} 7$.

Les consommations de pollen les plus importantes effectuées par les abeilles témoins s'établissent ainsi :

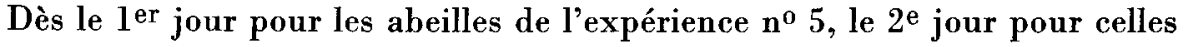
de l'expérience $\mathbf{n}^{0} 4$, le $3^{\mathrm{e}}$ jour pour les abeilles des expériences $\mathrm{n}^{\mathrm{os}} 1,2,6$. (Notons toutefois que les abeilles de l'expérience $\mathrm{n}^{\circ} 2$ ont consommé autant le $1^{\text {er }}$ et le $3^{\mathrm{e}}$ jour).

Les abeilles provenant de ruches réactivées réalisent leurs plus forts 
prélèvements un peu plus tard : le $4^{\mathrm{e}}$ jour pour l'expérience $n^{\circ} 3$, les $4^{\mathrm{e}}$ et $6^{\mathrm{e}}$ jours pour l'expérience $n^{0} 7$.

Les ingestions les plus remarquables sont effectuées par les témoins, comme par les traitées, par des abeilles d'été et d'automne, au cours des 3 premiers jours. L'âge des abeilles s'échelonne entre 1 et 5 jours.

Les abeilles provenant de ruches réactivées en hiver consomment moins, et leurs meilleures ingestions sont plus tardives.

La quantité totale de pollen ingéré s'avère toujours plus élevée chez les témoins. Toutefois les abeilles traitées paraissent prélever le pollen avec plus de régularité dans la plupart des cas.

\section{Comportement constructeur des abeilles.}

Pour essayer de mieux comprendre les fluctuations de la prise de nourriture, nous avons observé l'étirage puis la construction à partir de languettes de cire fixées au toit des cagettes, et mises à la disposition des abeilles.

Sur les 7 expériences réalisées, 5 d'entre elles donnèrent lieu à des constructions (Exp. $\left.\mathrm{n}^{\mathrm{os}} 1,2,4,5,6\right)$.

Chez les abeilles accompagnatrices de jeunes reines vierges, les premiers travaux d'étirage apparaissent entre le $3^{\mathrm{e}}$ et le $7^{\mathrm{e}}$ jour, les abeilles étant âgées de 3 à 8 jours. A la fin des expériences, les cires sont bien construites, le stockage de sirop abondant.

Dans le cas des abeilles en présence de jeunes reines fécondes, l'étirage commence le $6^{\mathrm{e}}$ ou le $7^{\mathrm{e}}$ jour, les abeilles étant âgées de 7 à 8 jours. Après 15 jours, les cires sont en général bien construites. Par contre le stockage varie selon les cas. Il est faible au cours de l'expérience $n^{0} 4$, un peu plus accentué pour l'expérience $n^{0} 5$, et en fin très important dans l'expérience $n^{\circ} 6$. A signaler aussi la ponte de nombreux œufs par les reines.

Les expériences effectuées avec des reines vieillies accompagnées de jeunes abeilles provenant de ruches réactivées, ne donnent lieu à aucune construction.

Les ouvrières témoins n'ont pas étiré, excepté celles de l'expérience $\mathbf{n}^{\mathbf{0}} \mathbf{1}$ qui se sont livrées au travail de la cire ont construit des cellules pour y stocker ensuite de la nourriture sucrée et ont même édifié des cellules royales dans lesquelles elles ont pondu.

D'après ces observations, nous considérons qu'en début d'expérience les ouvrières en présence de reines étirent, puis à partir du $7 \mathrm{e}$ jour d'encagement construisent. Des expériences sont actuellement en cours sur ce sujet. Ainsi entre le $1^{\mathrm{er}}$ et le $7^{\mathrm{e}}$ jour nous pouvons parler de consommation soit de pollen soit de candi. Mais après le $7^{\mathrm{e}}$ jour, dans certaines cagettes, la nourriture sucrée commence déjà à être stockée au fond de cellules étirées. Nous voyons donc apparaître un nouvel élément agissant sur la prise de nourriture sucrée : le stockage. 
Les abeilles d'été (Exp. $\mathrm{n}^{\text {os }} 1$ et 2) semblent construire plus volontiers que les abeilles d'automne $\left(\operatorname{Exp} . \mathrm{n}^{\text {os }} 4,5,6\right)$, et cela même en l'absence de reine (cas de l'expérience $\mathrm{n}^{\mathrm{0}} 1$ ). Toutefois dans des expériences précédentes (Roger, PaIN, 1966) nous avions obtenu des constructions en automne; les cagettes contenaient alors 100 ouvrières. Signalons aussi que la phase de construction débute pendant ou juste après un prélèvement important de nourriture.

\section{Taux de Mortalité.}

Nos expériences n'ont pas dépassé 15 jours pour plusieurs raisons. D'une part nous savons que l'état physiologique des abeilles encagées s'altère au fur et à mesure de leur vieillissement. D'autre part, le taux de mortalité croît rapidement au-delà du $15^{\mathrm{e}}$ jour de claustration. Par contre les abeilles supportent aisément une captivité de cet ordre. En effet sur l'ensemble des 7 expériences totalisant 5800 jeunes abeilles, le pourcentage total de mortalité s'est avéré très faible : $1,63 \%$, soit 95 abeilles (72 abeilles témoins et 23 traitées). Nous remarquerons que la mortalité est plus accentuée chez les témoins, ce qui confirme nos résultats antérieurs (Roger, PaIN, 1966).

\section{DISCUSSION}

Nous savons que les reines prolongent dans une certaine mesure la vie des ouvrières claustrées avec elles (Roger, Paiv, 1966).

En ce qui concerne les besoins alimentaires de la reine, ils sont d'après Chauvin (1965) de $14 \mathrm{mg}$ par jour, soit environ 5 grammes pour une année. Cette consommation est trop faible pour avoir exercé une influence sur les résultats que nous avons obtenus.

D'autre part, un rôle primordial est attribué à la reine dans le comportement de construction des ouvrières : Darchen (1960), Chauvin, Darchen, Pain (1961). Comme nous venons de le voir, nous avons obtenu de nombreuses constructions au cours de nos expériences, principalement avec les abeilles accompagnatrices, mais aussi avec des abeilles orphelines (Exp. $n^{\circ} 1$ ). C'est entre le $6^{\mathrm{e}}$ jour et le $7^{\mathrm{e}}$ jour de vie que les abeilles construisent, ce qui correspond aux données de Darchen (1957). Si nous abordons ce problème, c'est qu'il intervient d'une façon directe dans nos expériences, et qu'il modifie le comportement alimentaire des abeilles. En effet, dans la plupart des cas, les abeilles manifestent un ralentissement dans la prise de nourriture sucrée à compter du $6^{\mathrm{e}}$ jour et jusqu'au $12^{\mathrm{e}}$ jour. Donc au cours d'une première période, les abeilles consomment de fortes quantités de nourriture. Étant un peu plus âgées, leurs prélèvements de candi diminuent pendant la période d'étirage 
et de début de construction. Dans une deuxième période, les abeilles prélèvent de nouveau beaucoup de nourriture, ce qui semblerait coïncider avec l'apparition du stockage.

La consommation de pollen est surtout très marquée pendant les 4 à 5 premiers jours de vie des jeunes abeilles. Celle de sucre augmente progressivement jusqu'au $10^{\mathrm{e}}$ jour (PAIN, 1961). Nos résultats vont dans le même sens puisque les prélèvements importants communs à toutes nos expériences se situent pour le candi, d'une part les $3^{\mathrm{e}}$ et $6^{\mathrm{e}}$ jours, d'autre part entre les $9^{\mathrm{e}}$ et $12^{\mathrm{e}}$ jours. La consommation de pollen s'avère importante entre les $1^{\mathrm{er}}$ et $6^{\mathrm{e}}$ jours d'encagement, selon les expériences. Ainsi les prélèvements des deux nourritures s'effectuent selon des modalités différentes.

Il nous a semblé intéressant de grouper les prélèvements de nourriture sucrée selon la nature des reines : d'une part les 2 expériences comprenant de jeunes reines vierges ( $n^{0 s} 1$ et 2 ), d'autre part les 3 expériences réalisées avec de jeunes reines fécondes $\left(n^{08} 4,5,6\right)$.

Si nous examinons les résultats globaux obtenus de cette manière, il apparaît que les quantités de nourriture prélevées par les abeilles des deux lots ainsi formés sont comparables. Ainsi, nous obtenons après 15 jours d'expérimentation, pour les abeilles témoins des expériences $\mathrm{n}^{\circ} 1+2: 213,7 \mathrm{mg}$ et $212 \mathrm{mg} / \mathrm{ab}$ pour les expériences $\mathrm{n}^{08} 4+5+6$. La prise de nourriture moyenne quotidienne ainsi calculée s'élève respectivement à 14,2 et 14,1 mg/ab.

Pour les abeilles en présence de jeunes reines, les chiffres sont plus élevés que chez les témoins et pratiquement identiques soit : 278,2 et $276,9 \mathrm{mg} / \mathrm{ab}$ ou encore $18,5 \mathrm{mg} / \mathrm{ab} / \mathrm{jour}$. Il semble donc que les reines ont influé sur le prélèvement de candi dans les mêmes proportions, qu'elles soient vierges ou fécondes. Notons toutefois que chez les reines fécondes les prélèvements présentent plus d'homogénéité (et varient de 13,3 à $21,2 \mathrm{mg} / \mathrm{ab} /$ jour). Par contre, chez les reines vierges, ces prélèvements sont plus irréguliers et oscillent de 8,9 à $26,9 \mathrm{mg} / \mathrm{ab} /$ jour. Les quantités de nourriture prélevées par les témoins ont atteint un certain seuil représentant les besoins alimentaires des abeilles orphelines. Ce seuil n'apparaît pas chez les ouvrières traitées, car elles ont la possibilité d'emmagasiner de la nourriture alors que les ouvrières témoins ne stockent pas. La capacité du jabot étant relativement constante (KELLogG, 1958), elles ne peuvent absorber davantage de nourriture.

Par contre, les valeurs totales obtenues au cours des expériences n ${ }^{\text {os }} 3$ et 7 chez les abeilles témoins issues de ruches réactivées, paraissent moins homogènes lorsque nous les comparons : 227,7 et $245,4 \mathrm{mg} / \mathrm{ab}$.

Pour des abeilles de même origine, mais en présence de reines vieillies, les prélèvements restent inférieurs à ceux effectués par les témoins : 202,1 et $218,3 \mathrm{mg} / \mathrm{ab}$. Ces chiffres se rapprochent davantage de ceux obtenus pour les abeilles orphelines des expériences avec jeunes reines vierges ou fécondes. 
Le taux de consommation est de 18,2 mg/ab selon Sitbon (1967), pour des abeilles d'Hiver groupées orphelines et d'âge indéterminé. Or nous obtenons le même chiffre : $18,5 \mathrm{mg} / \mathrm{ab}$ mais pour de jeunes abeilles en présence de reine.

Abstraction faite des méthodes de contrôle de la nourriture et du temps d'expérimentation, Maurizio (1945) donne les chiffres suivants : 14-16 mg pour de jeunes abeilles d'été ou d'hiver, 20-26 mg pour de vieilles abeilles d'âge indéterminé. DE Groot (1953) indique : 12,2 et $12,7 \mathrm{mg} / \mathrm{ab}$ pour de jeunes abeilles orphelines, et 29,2 et $35,2 \mathrm{mg} / \mathrm{ab}$ pour de vieilles abeilles orphelines.

En ce qui concerne le pollen, nous avons vu que les abeilles témoins en consomment toujours davantage que les abeilles traitées, cependant les résultats ne sont jamais significatifs. WAHL (1963) conclut après avoir contrôlé la consommation de pollen dans de petites colonies de 150 abeilles pourvues de reine, que la consommation pollinique n'est pas plus importante dans des colonies contenant une reine que dans les colonies orphelines.

\section{CONCLUSION}

Lors d'expériences précédentes nous avons démontré que la présence de reines vivantes vierges ou fécondes, jeunes ou vieillies, ou bien de reines mortes abaisse à des degrés divers le taux de mortalité de petits groupes de 50 à 100 ouvrières.

De même, nous mettons ici en évidence l'influence exercée par les jeunes reines vierges ou fécondes sur la prise de nourriture sucrée. Cependant l'âge de la reine, le temps passé en cagette avant l'expérimentation, l'état physiologique des abeilles sont des facteurs importants capables de modifier profondément les résultats.

Ainsi les abeilles accompagnatrices de très jeunes reines vierges (8 jours de vie et de claustration) prélèvent légèrement plus de candi que les témoins.

Nous obtenons des résultats identiques pour des abeilles en présence de jeunes reines fécondes.

Les chiffres obtenus avec des reines présentant peu de jours d'encagement ne donnent pas des valeurs statistiquement significatives.

Par contre, des reines vierges âgées de 18 jours et des reines fécondes ayant séjourné de 20 à 34 jours en cagette donnent des résultats hautement significatifs.

Enfin, nous obtenons avec des reines ayant vécu plus de 3 mois en cagettes, des résultats différents. Les témoins prélèvent davantage de nourriture sucrée, mais les valeurs obtenues ne sont pas significatives. 
Or dans nos expériences relatives à l'étude du taux de mortalité, les reines vieillies abaissaient la mortalité de leurs abeilles accompagnatrices dans des proportions significatives.

Les facteurs influençant soit la mortalité, soit la prise de nourriture, interviennent de façon différente. Ces données nous permettent de mettre l'accent sur une corrélation possible existant d'une part entre l'âge des reines et d'autre part la mortalité et la prise de nourriture sucrée.

Les périodes de forte consommation de sucre se situent pour toutes les abeilles accompagnées de reine, entre le $2^{\mathrm{e}}$ et le $4^{\mathrm{e}}$ jour d'expérience. Les ouvrières sont alors âgées de 3 à 5 jours.

Pour les témoins, cette première période de fort prélèvement de candi, se situe un peu plus tard, vers le $6^{\mathrm{e}}$ jour.

Une deuxième période de forts prélèvements apparaît entre le $9 \mathrm{e}$ et le $12^{\mathrm{e}}$ jour chez les abeilles en présence de jeunes reines, et un peu plus tardivement avec les reines vieillies.

Cette seconde période intervient entre le $9^{\mathrm{e}}$ et $\mathrm{le} 13^{\mathrm{e}}$ jour chez les abeilles orphelines et semble être influencé par l'état physiologique de ces abeilles, sans doute différent selon la saison.

Pour le pollen les consommations les plus élevées sont réalisées pour les les témoins comme pour les traitées par les abeilles d'été et d'automne au cours des 3 premiers jours, l'âge des abeilles s'échelonnant entre 1 et 5 jours.

Les abeilles provenant de ruches ( réactivées ) consomment moins de pollen et les ingestions les plus importantes sont enregistrées du $4^{\mathrm{e}}$ au $7^{\mathrm{e}}$ jour de vie.

Dans l'ensemble, le pollen est consommé par les abeilles traitées avec plus de régularité.

Chez la jeune abeille le $4^{\mathrm{e}}$ jour paraît jouer un très grand rôle du point de vue de la nutrition. D'ailleurs Pershad (1967) met en évidence l'influence très nette de l'âge de l'abeille sur l'intensité des échanges alimentaires. Les abeilles donneuses et receveuses de même âge ont des échanges maximum vers le $4^{\mathrm{e}}$ jour.

Nos observations nous ont amené à tenir compte du comportement de construction. En effet, les travaux d'étirage apparaissent entre le $3^{\mathrm{e}}$ et le $7^{\mathrm{e}}$ jour dans les populations contenant une reine. La nourriture sucrée commence à être stockée au fond de cellules préalablement étirées par les abeilles, après le $7^{\mathrm{e}}$ jour.

A la consommation s'ajoute donc un autre facteur, le transport de nourriture. C'est la raison pour laquelle nous avons analysé les résultats de chaque expérience entre le $1^{\mathrm{er}}$ et le $7^{\mathrm{e}}$ jour et entre le $1^{\mathrm{er}}$ et le $15^{\mathrm{e}}$ jour.

Cette étude, une fois de plus, montre l'influence exercée par la reine sur les 
abeilles qui l'entourent. La reine conditionne le développement de divers organes de l'ouvrière, la construction cirière, le taux de mortalité et la prise de nourriture.

Il nous paraît donc intéressant, dans le cadre des interactions entre reines et ouvrières, de poursuivre l'étude des divers facteurs agissant sur le déterminisme de la prise de nourriture des abeilles encagées.

Reçu pour publication en mars 1971.

Eingegangen im März 1971.

\section{ZUSAMMENFASSUNG}

Die unterschiedliche Nahrungsaufnahme (Pollen und Zuckerteig) junger Bienen in Abhängigkeit von der Anwesenheit unbegatteter, begatteter, junger oder alter Königinnen worde untersucht.

Die Versuchsbienen schlüpften bei $32^{\circ} \mathrm{C}$ im Brutschrank und wurden bei den gleichen Bedingungen gehalten. Die jungen Arbeiterinnen bekannten Alters wurden in Gruppen von 50 Individuen in kleinen, mit einer Mittelwand ausgestatteten Käfigen nach PalN (1966) gehalten. Jede Versuchsreihe umfasste mindestens zwei Gruppen von sechs Käfigen. Die Völkchen der einen Gruppe blieben weisellos, sie dienten als Kontrollen, die der anderen Gruppe, die eigentlichen Versuchsvölkchen, wurden beweiselt.

Wir benutzten Königinnen bekannten Alters verschiedener Art :

junge ( 7 - 18 tägige) unbegattete 우 (Versuch 1 u. 2)

im Brutschrank gealterte, unbegattete $\circ \circ$, zu Beginn des Versuchs 165 Tage alt (Versuch 3)

junge begattete $\$ 9$, die 44-51 Tage in Dreiwaben-Kästchen im Freien gehalten worden waren und dann 6-34. Tage im Versuchskäfig lebten (Versuch 4, 5 u. 6)

Alte, begattete, im Brutschrank gehaltene §o; 138 Tage alt (Versuch 7).

Die Königinnen der verschiedenen Versuchsreihen waren Geschwister, in Bures-surYvette in Italienervölkern gezogen.

Die aufgenommenen zucker- und pollenhaltigen Nahrungsmengen wurden getrennt gewichtsmässig bestimmt und täglich zur gleichen Zeit gewogen, und zwar die Futterteigmengen 14 Tage lang, der Pollen 8 Tage. Der Feuchtigkeitsverlust der Nahrung wurde berücksichtigt. Die statistische Auswertung der Ergebnisse erfolgte nach dem F-Test von SNEDEcor.

In allen Fällen nahmen die Begleitbienen junger Königinnen mehr zuckerhaltige Nahrung auf als weisellose Bienen. Die statistischen Werte sind für die Versuche 2,5 und 6 besonders beweiskräftig. Wir konnten ausserdem beobachten, dass das Alter der Königinnen, die Zeit, die sie vor Versuchsbeginn im Käfig verbrachten und der physiologische Zustand der Bienen ebenso wichtige, die Ergebnisse beeinflussende Faktoren sind.

So haben wir bei Zusammenfassung der Daten analoger Versuchsreihen eine durchschnittliche Zuckerteigaufnahme von $14 \mathrm{mg}$ je Biene und Tag bei den weisellosen Kontrollen und 18,5 mg je Biene und Tag bei den Völkehen mit jungen unbegatteten oder begatteten Königinnen festgestellt. Dagegen nahmen die Begleitbienen alter Königinnen (Versuch 3 und 7) geringere Futtermengen auf, nämlich $13,5 \mathrm{mg}$ je Biene und Tag, während bei den weisellosen Arbeiterinnen auch hier 15,2 und $16,4 \mathrm{mg}$ je Biene und Tag registriert wurden.

Die ermittelten Werte sind bei weisellosen Arbeiterinnen denen von MaUrizio und DE Groot und neuerdings von $W_{A H L}$ und StтвоN angegebenen Werten vergleichbar. 
Die Kurvenanalyse zeigte, dass die Arbeiterinnen im Beisein junger unbegatteter oder begatteter Königinnen vom 3. Tag ab grössere Futtermengen rascher aufnahmen, die Kontrollbienen dagegen erst vom 6. Tag ab. Eine erneute Zunahme des Futterverbrauchs vom 9.15. Tag konnte in beiden Bienengruppen festgestellt werden, allerdings, je nach Versuch, mit mehr oder minder bedeutenden Abweichungen.

In bezug auf den Pollen waren bei den weisellosen Bienen (F-Test positiv für Versuch 6) die aufgenommenen Mengen stets grösser; ihr täglicher Durchschnittsverbrauch betrug 4,5 mg bis 4,7 mg je Biene und Tag, während für die Versuchsbienen ein Verbrauch von $3,2 \mathrm{mg}$ - 3,6 $\mathrm{mg}$ je Biene und Tag registriert wurde.

Andererseits wurde der stärkste Futterverbrauch bei den Kontrollen ebenso wie bei den Versuchstieren bei Sommer- und Herbstbienen während der ersten drei Versuchstage registriert. Dagegen verbrauchten Arbeiterinnen, die aus im Winter reaktivierten Völkern stammten, weniger Pollen; ihr Höchstverbrauch tritt erst später auf.

Im Verlauf dieser Versuche konnten noch andere Beobachtungen gemacht werden : Die Königin ist von primärer Bedeutung für das Bauverhalten der Arbeiterinnen; selbst im Versuchskäfig bauten die Begleitbienen. Sie bauten zwischen dem 6. und 7. Lebenstag, was mit den Angaben von Darchen (1957) übereinstimmt. Sind die Zellen erst einmal gebaut, wird die zuckerhaltige Nahrung auch von den Bienen gespeichert.

Nach unseren Beobachtungen würden die Futtervorräte in den Zellen dem tatsächlichen Nahrungsbedarf nicht entsprechen. Es handelt sich hier eher um ein Verhalten, das LouvEAUX in bezug auf die Pollenversorgung eines Volkes als “ hoarding 》 bezeichnet.

Einmal mehr konnte nachgewiesen werden, dass zwischen Königin und Arbeiterinnen wechselseitige Beziehungen bestehen, die in $Z$ wergvölkchen untersucht werden können. Die Königinnen beeinflussen auf bestimmte Weise die Sterblichkeitsquote und die Nahrungsaufnahme der sie umgebenden Bienen.

In einer weiteren Arbeit sollen diese Untersuchungen fortgesetzt werden.

\section{REFERENCES BIBLIOGRAPHIQUES}

Chauvin R., 1968. Traité de biologie de l'Abeille. Éd. Masson et Cie. Paris, t. I, p. 372.

Chauviv R., Darchen R., Pain J., 1961. Sur l'existence d'une hormone de construction chez les abeilles, C. R. Acad. Sci., Paris, 253, 1135-1136.

Darchen R., 1957. La reine d'Apis mellifica, les ouvrières pondeuses et les constructions cirières, Insectes Sociaux, 4 (1), 322-325.

Darchen R., 1960. Les régulations neurohormonales de l'instinct constructeur des ouvrières d'Apis mellifica, Ann. Abeille, 3 (4), 329-333.

Delvent-Salleron F., 1963. Étude des échanges de nourriture entre reines, mâles et ouvrières d'Apis mellifica L., Ann. Abeille, 6 (1), 201-227.

De Groot A. P., 1953. Protein and amino acid requirements of the honeybee, Physiol. Comp. Oecol., 3, 197-285.

Kellogg G. R., 1958. Honey sac capacity in some honeybees, Amer. Bee J., 98, p. 101.

LEgay J.-M., 1957. La prise de nourriture chez le ver à soie. Ann. Epiphyties.

Louveaux J., 1958. Recherches sur la récolte de Pollen par les abeilles (Apis mellifica L), Thèse Sci. Nat., Paris, ${ }^{\circ} 3997,1-206$.

Maunizio A., 1945. Trachtkrankheiten der Bienen : I-Vergiftungen bei einseitiger Tracht von Rosskastanien., Beih. Schweiz. Bienenztg., 1 (8), 337-368.

Maunizio A., 1946. Beobachtungen über die Lebensdauer und den Futterverbrauch gefangen gehaltener Bienen, Beih. Schweiz. Bienenztg., 2 (13), 1-44. 
PaIN J., 1961. Sur quelques facteurs alimentaires accélérateurs du développement des œufs dans les ovaires des ouvrières d'abeilles (Apis m. L.), Insectes sociaux, 8 (1), 31-93.

Pain J., 1963. L'alimentation de la jeune abeille, Ann. Nutrit. Aliment., 17 (1), 307-312.

PaIN J., 1966. Nouveau modèle de cagettes expérimentales pour le maintien d'abeilles en captivité, Ann. Abeille, 9 (1), 71-76.

Pain J., Maugenet J., 1966. Recherches biochimiques et physiologiques sur le pollen emmagasiné par les abeilles, Ann. Abeille, 9 (3), 209-236.

Pershad S., 1967. Analyse de différents facteurs conditionnant les échanges alimentaires dans une colonie d'abeilles Apis mellifica $L$. au moyen du radio-isotope $\mathrm{P} 32, A n n$. Abeille, 10 (3), 139-197.

Roger B., Pain J., 1966. L'influence de la reine d'abeille (Apis mellifica L.) sur le taux de mortalité des ouvrières accompagnatrices, Ann. Abeille, 9 (1), 5-36.

Sitbon G., 1967. L'effet de groupe chez l'abeille : 1 - L'abeille d'hiver; survie et consommation de candi des abeilles isolées ou groupées, Ann. Abeille, 10 (2), 67-82.

WAHL O., 1963. Vergleichende Untersuchungen über den Nährwert von Pollen, Hefe, Sojamehl und Trockenmilch für die Honigbiene. Zeitschr. $f$. Bienenf., 6 (8), 209-280. 